\title{
Modeling Geometric Deformations in EPI Time Series
}

\author{
J esper L. R. Andersson, ${ }^{1}$ Chloe Hutton, J ohn Ashburner, Robert Turner, and Karl Friston \\ The Wellcome Department of Cognitive Neurology, London, United Kingdom
}

Received J une 13, 2000

\begin{abstract}
Even after realignment there is residual movementrelated variance present in $\mathrm{fMRI}$ time-series, causing loss of sensitivity and, potentially, also specificity. One cause is the differential deformation of the sampling matrix, by field inhomogeneities, at different object positions, i.e., a movement-by-inhomogeneity interaction. This has been addressed previously by using empirical field measurements. In the present paper we suggest a forward model of how data is affected by an inhomogeneous field at different object positions. From this model we derive a method to solve the inverse problem of estimating the field inhomogeneities and their derivatives with respect to object position, directly from the EPI data and estimated realignment parameters. The field is modeled as a linear combination of cosine basis fields, which facilitates a fast way of implementing the necessary matrix operations. Simulations suggest that the solution is tractable and that the fields are estimable given the deformed images and knowledge of the relative positions at which they have been acquired. An experiment on a subject performing voluntary movements in the scanner yielded plausible estimates of the deformation fields and their application to "unwarp" the time series significantly reduced movement-related variance. 02001 Academic Press
\end{abstract}

\section{INTRODUCTION}

Subject movement can be a serious confound when assessing brain function from BOLD changes in EPI images (Hajnal et al., 1994). Consequently, numerous methods for retrospective realignment of serial functional MR images have been developed (Friston et al., 1995; Hajnal et al., 1995; J iang et al., 1995; Woods et al., 1998) where the accuracy of estimated registration parameters is in the subvoxel range. However, even after registration considerable motion-related variance

\footnotetext{
${ }^{1}$ To whom correspondence and reprint requests should be addressed at present address: Karolinska MR Research Center, Karolinska Hospital N-8, 17176 Stockholm, Sweden. Fax: 46851776111. E-mail: jesper@mrc.ks.se.
}

remains in the data (Friston et al., 1996), leading to decreased sensitivity and, in the case of task-correlated motion, specificity. Several explanations have been suggested for this residual variance including interpolation errors (Ostuni et al., 1997; Grootoonk et al., 2000), which has lead to the development of fast algorithms for Fourier interpolation (E ddy et al., 1996; Cox and J esmanowicz, 1999), and spin-history effects (Friston et al., 1996). Another possible source is the interaction between susceptibility-induced distortions and subject movement (Wu et al., 1997; J ezzard and Clare, 1999). Briefly, magnetization inhomogeneities cause geometric distortions which are appreciable mainly in the phase encoding direction, and these distortions may change as the subject moves in the scanner. Methods have been developed to correct for these effects by explicitly measuring field inhomogeneity (J ezzard and Balaban, 1995; Reber et al., 1998; Chen and Wyrwicz, 1999). The direct method of measuring field maps is associated with practical difficulties due to measure ment noise (uncertainty of the field maps) and rapid loss of signal toward the edges of the object, leading to difficulties in unwrapping the phase. In addition, if the objective is to eliminate/reduce the distortion by move ment interactions, then a field map has to be generated for each scan in the time-series.

In the present paper we suggest generative or forward models for how the images are affected by movement in the presence of an inhomogeneous field. From these we derive a method for solving the inverse problem to estimate the deformation field directly from the EPI images. We present a mathematical framework for the models and assess them using simulated and experimental data. This paper is concerned with the mathematical and theoretical basis of our approach. A subsequent paper will address validity and empirical applications.

\section{THEORY}

In this section we will present generative forward models for three specific cases of movement-by-inhomogeneity interactions. These cases are (i) inhomoge- 
neities fixed with respect to the object, (ii) inhomogeneities fixed with respect to the scanner, and (iii) inhomogeneities that change with subject movement. We will derive expressions for solving the corresponding inverse problems, which yield estimates of the deformation fields.

It will be demonstrated that the third model encompasses both the former models as special cases. It can therefore be considered as a general model.

\section{Rigid Body I mage Registration}

An image can be considered as a scalar function of a vector argument such that $f_{i}(\mathbf{x})$ denotes the intensity of the ith image in a time-series at the location $\mathbf{x}=[\mathbf{x} y$ $\mathbf{z}]^{\top}$. L et $\mathbf{T}(\mathbf{p})$ denote a $4 \times 4$ rigid body transformation matrix mapping a location $\mathbf{x}$ to a new location $\mathbf{x}^{\prime}$ through $\left[\mathbf{x}^{\prime \top} 1\right]^{\top}=\left[\begin{array}{lll}x^{\prime} y^{\prime} z^{\prime} & 1\end{array}\right]^{\top}=\mathbf{T}\left[\begin{array}{ll}\mathbf{x}^{\top} & 1\end{array}\right]^{\top}$, where $\mathbf{T}$ is parameterized through the vector $\mathbf{p}$ and contains three rotation angles and three translations. Realignment proceeds by aligning all scans to some reference scan, often the first. It involves estimating $\mathbf{p}$ for each scan by modeling the difference between the reference scan and the scan to realign using the first two terms of a Taylor expansion

$$
\mathrm{f}_{1}(\mathbf{x}) \approx \mathrm{f}_{\mathrm{i}}(\mathbf{x})+\left[\frac{\partial \mathrm{f}_{1}}{\partial \mathrm{p}_{1}}(\mathbf{x}) \frac{\partial \mathrm{f}_{1}}{\partial \mathrm{p}_{2}}(\mathbf{x}) \cdots \frac{\partial \mathrm{f}_{1}}{\partial \mathrm{p}_{6}}(\mathbf{x})\right] \mathbf{p}_{\mathrm{i}}
$$

$\mathbf{p}_{\mathrm{i}}$ determines the transformation $\mathrm{T}$ such that $\mathrm{f}_{1}(\mathbf{x}) \approx$ $\mathrm{f}_{\mathrm{i}}\left(\mathbf{T}\left(\mathbf{p}_{\mathrm{i}}\right) \mathbf{x}\right)$. By extending the equation above to all $\mathrm{n}$ voxels a least squares estimate of $\mathbf{p}$ obtains $\hat{\mathbf{p}}_{\mathrm{i}}=$ $\left(\mathbf{A}^{\top} \mathbf{A}\right)^{-1} \mathbf{A}^{\top}\left(\mathbf{f}_{1}-\mathbf{f}_{\mathrm{i}}\right)$, where $\mathbf{A}$ is an $\mathrm{n} \times 6$ matrix containing the partial derivatives of $f_{1}, \mathbf{f}_{1}$ and $\mathbf{f}_{\mathrm{i}}$ are $n \times 1$ column vectors containing intensity values for the first and ith image at all voxels. I terative estimates for $\mathbf{p}_{i}$ can be obtained with high accuracy (Ashburner and Friston, 1999a). $\mathbf{T}_{\mathrm{i}}$ now maps the coordinates in the first image to the coordinates in the ith, i.e., $f_{1}(\mathbf{x}) \approx$ $f_{i}\left(T_{i} \mathbf{x}\right)$. We denote the matrix consisting of the upper left $3 \times 3$ partition of $\mathbf{T}_{\mathrm{i}}$, embodying rotation alone, by $\mathbf{R}_{\mathrm{i}}$.

\section{Geometric Deformations of Echoplanar Images}

In the formation of echo planar images (EPI) position is given by frequency, which is in turn determined by local field strength. For a perfectly homogenous field, combined with a perfectly linear gradient, this means that an inverse F ourier transform of the acquired data yields an image of the object sampled on a regular grid. In the presence of field inhomogeneities, e.g., caused by poor shimming or by an object causing local inhomogeneities, frequency will not change linearly with position, and geometric distortions will ensue. Due to the relatively long time during which frequency errors are allowed to develop into phase errors, these distortions are much larger in the phase encoding direction (for EPI), and negligible in the frequency encoding direction (J ezzard and Balaban, 1995). Therefore an image intensity value, that we think represents $f(x)$, really represents $f(x+\mathbf{d}(\mathbf{x}))=f\left([x y z]^{\top}+[0 d(x, y, z) 0]^{\top}\right)$, where the value of $\mathbf{d}(\mathbf{x})$ is proportional to the field inhomogeneity at $\mathbf{x}$. We have assumed here that the phase encoding direction coincides with the y-direction (anterior-posterior). Because the $x$ - and z-components of $\mathbf{d}(\mathbf{x})$ are zero it might seem reasonable to represent it by a scalar valued function of a vector (i.e., $d(\mathbf{x})$ ) but, for reasons that will become clear later, we represent it as a vector field. Henceforth we will use the terms field inhomogeneities and deformation fields interchangeably.

\section{A Model for Inhomogeneities Fixed with Respect to Object (Model 1)}

In this paper we consider three different models for how a deformation field affects data in the context of subject movements. These models correspond to three distinct cases with respect to the behaviour of the deformations when the object moves. The first case is when the deformation field moves perfectly with the object, a case we would expect to occur when the deformations are caused by the object itself and where rotations only occurred around an axis parallel to the magnetic field. Now consider two images $f_{1}$ and $f_{2}$ such that $\mathbf{T}$ maps $f_{2}$ onto $f_{1}$. Then we have effectively sampled the object at positions given by the arguments of

$$
\mathrm{f}_{1}(\mathbf{X}+\mathbf{d}(\mathbf{X}))
$$

for the stationary image and

$$
\mathrm{f}_{2}\left(\mathbf{T}^{-1} \mathbf{X}+\mathbf{R}^{-1} \mathbf{d}\left(\mathbf{T}^{-1} \mathbf{X}\right)\right)
$$

for the moved image. Here $\mathbf{X}=\left[\begin{array}{ll}\mathbf{x}_{1}^{\top} & 1\end{array}\right]^{\top}\left[\begin{array}{ll}\mathbf{x}_{2}^{\top} & 1\end{array}\right]^{\top} \ldots\left[\begin{array}{l}\mathbf{x}_{n}^{\top} \\ \hline\end{array}\right.$ $\left.1]^{\top}\right]$ denotes the one-extended $4 \times \mathrm{n}$ matrix containing the coordinates of all voxel centres in an object fixed system, i.e., a system defined relative to the object in the referencescan. $\mathbf{R}$ denotes the rotation part of $\mathbf{T}$ and $\mathbf{d}(\mathbf{X})$ is defined in the frame of $\mathrm{f}_{1}$. Assuming we know $\mathbf{T}$ (or rather an estimate from rigid body realignment), we can use it to realign $\mathrm{f}_{2}$ yielding

$$
\mathrm{f}_{2}\left(\mathbf{T T}^{-1} \mathbf{X}+\mathbf{R}^{-1} \mathbf{d}\left(\mathbf{T T}^{-1} \mathbf{X}\right)\right)=\mathrm{f}_{2}\left(\mathbf{X}+\mathbf{R}^{-1} \mathbf{d}(\mathbf{X})\right),
$$

which should be compared to Eq. (1) for the stationary image. Hence, despite the deformation field moving perfectly with the object, there will still be deformation-related differences after realignment. 

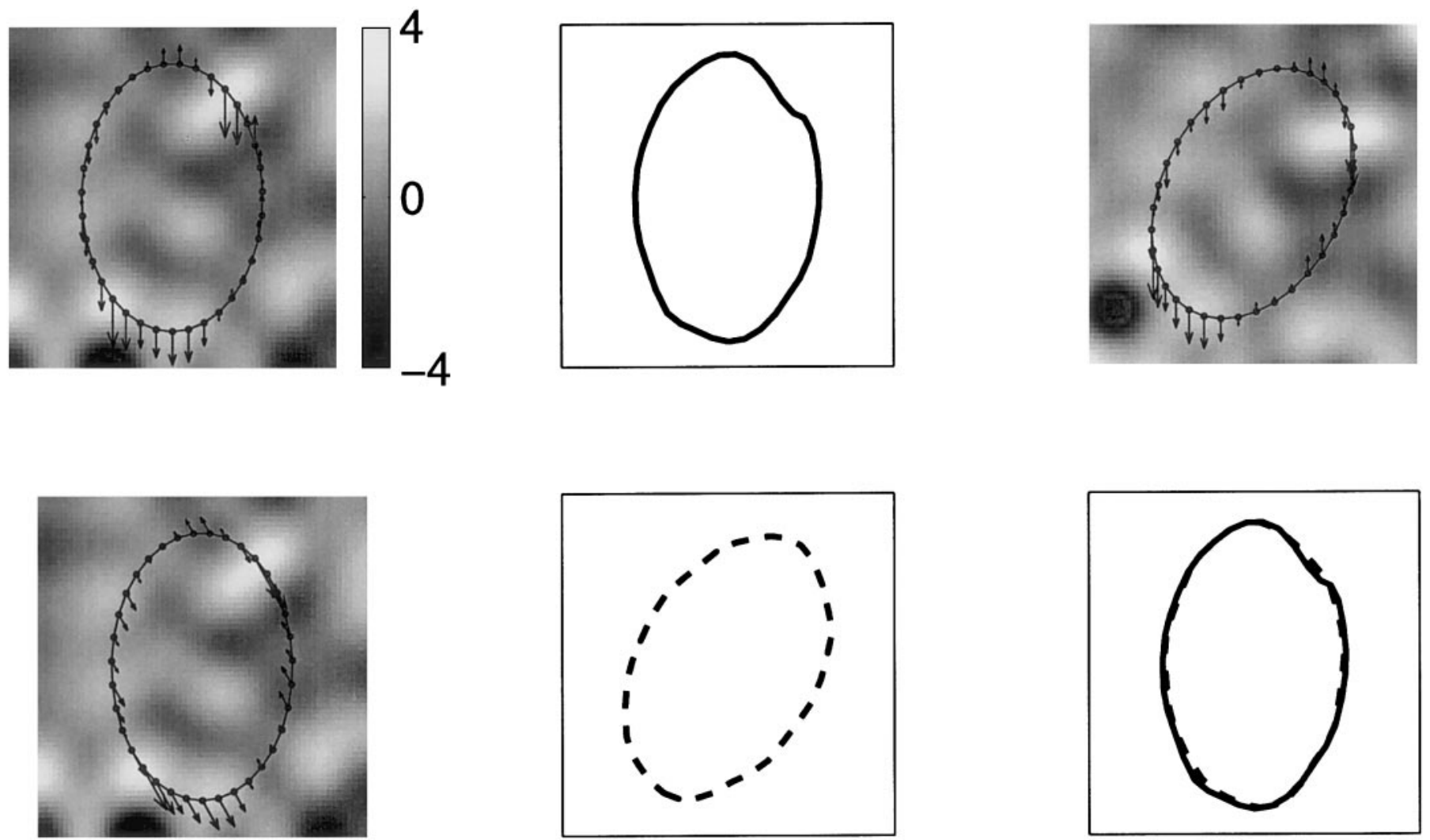

FIG. 1. Graphical explanation of the forward model defined by Eqs. (1) and (3). Upper left panel: shows an example of a random deformation field. The grey values indicate the deviation from a regular grid when sampling an object, where light shades indicate sampling points have been deflected upwards and dark shades indicate downwards deflection. The color bar is graded in units of voxels. The contour indicates a brain-like shape and the arrows show the direction and magnitude of apparent deformation when imaged in this deformation field. U pper middle panel: shows the resulting deformed contour. U pper right panel: shows the object following a 30 rotation, where the deformation field has rotated together with the object. Note how each point on the contour is associated with an identical arrow as in the unrotated case. Lower left panel: shows the same situation in a realigned (object) framework (which is the framework our notation uses). It is now clear that although each point is affected by a deformation of identical magnitude, it is now acting in a different direction. This is caused by the phase encode direction being stationary with respect to the scanner framework rather than the object framework. Lower middle panel: shows the resulting deformed contour for the rotated case. Lower right panel: demonstrates the mismatch between two images of an identical object imaged in identical deformation fields, but where in one case the object had been rotated. Note that according to this model differential deformations will only be introduced by rotations (i.e., it is insensitive to translations).

Intuitively this can be seen by considering lying in the scanner looking up, where the deformations serve to stretch/compress the brain (or images thereof) in the anterior-posterior direction. Now consider rotating your head to look towards the side in the scanner. Although every voxel will be affected by a deformation of identical magnitude as before, it will now be stretching/compressing the brain in the right-left direction. Obviously, after realignment this will cause differences in the realigned images, since they will have been deformed in different directions. A graphical illustration is presented in Fig. 1.

Now assume an inverse deformation field exists, denoted $\mathbf{d}^{-1}$, such that $\mathbf{X}+\mathbf{d}(\mathbf{X})+\mathbf{d}^{-1}(\mathbf{X}+\mathbf{d}(\mathbf{X}))=\mathbf{X}$, which for small deformations will be reasonably ap- proximated by $-\mathbf{d}$. We seek to estimate the field $\hat{\mathbf{d}}^{-1}$ that minimizes the difference between $f_{1}(\mathbf{X}+\mathbf{d}(\mathbf{X})+$ $\left.\hat{\mathbf{d}}^{-1}(\mathbf{X})\right)$ and $\mathrm{f}_{2}\left(\mathbf{X}+\mathbf{R}^{-1} \mathbf{d}(\mathbf{X})+\mathbf{R}^{-1} \hat{\mathbf{d}}^{-1}(\mathbf{X})\right)$, where $\mathrm{f}_{2}$ is in a realigned framework (i.e., $\mathbf{X}=\mathbf{T} \mathbf{X}^{\prime}$, where $\mathbf{X}^{\prime}$ is the grid onto which $f_{2}$ was originally sampled). Assume the shape of $\hat{\mathbf{d}}^{-1}$ is known, but not its magnitude, i.e., that $\hat{\mathbf{d}}^{-1}=\beta \mathbf{b}$, where $\mathbf{b}$ is a known field and $\beta$ is an unknown scalar. We may now express $f_{1}\left(\mathbf{x}_{i}\right)$ and $f_{2}\left(\mathbf{x}_{i}\right)$ as a function of $\beta$ as the first two terms of a Taylor expansion such that

$f_{1}\left(\mathbf{x}_{i}, \beta\right)$

$$
\approx \mathrm{f}_{1}\left(\mathbf{x}_{\mathrm{i}}, 0\right)+\beta\left[\frac{\partial \mathrm{f}_{1}}{\partial \mathbf{x}}\left(\mathbf{x}_{\mathrm{i}}\right) \frac{\partial \mathrm{f}_{1}}{\partial \mathrm{y}}\left(\mathbf{x}_{\mathrm{i}}\right) \frac{\partial \mathrm{f}_{1}}{\partial \mathrm{z}}\left(\mathbf{x}_{\mathrm{i}}\right)\right]\left[\begin{array}{c}
0 \\
\mathrm{~b}\left(\mathbf{x}_{\mathrm{i}}\right) \\
0
\end{array}\right]
$$


and

$$
\begin{aligned}
& \mathrm{f}_{2}\left(\mathbf{x}_{i}, \beta\right) \\
& \approx \mathrm{f}_{2}\left(\mathbf{x}_{i}, 0\right)+\beta\left[\frac{\partial \mathrm{f}_{1}}{\partial \mathrm{x}}\left(\mathbf{x}_{\mathrm{i}}\right) \frac{\partial \mathrm{f}_{1}}{\partial \mathrm{y}}\left(\mathbf{x}_{\mathrm{i}}\right) \frac{\partial \mathrm{f}_{1}}{\partial z}\left(\mathbf{x}_{\mathrm{i}}\right)\right] \mathbf{R}^{-1}\left[\begin{array}{c}
0 \\
\mathrm{~b}\left(\mathbf{x}_{\mathrm{i}}\right) \\
0
\end{array}\right],
\end{aligned}
$$

where the derivative of $f_{1}$ is used in both expressions for computational convenience. Finding the $\beta$ for which $f_{1}\left(\mathbf{x}_{i}, \beta\right)=f_{2}\left(\mathbf{x}_{i}, \beta\right)$ means solving the equation

$$
\begin{aligned}
\mathrm{f}_{1}\left(\mathbf{x}_{\mathrm{i}}\right) & -\mathrm{f}_{2}\left(\mathbf{x}_{\mathrm{i}}\right) \\
& =\beta\left[\frac{\partial \mathrm{f}_{1}}{\partial \mathrm{x}}\left(\mathbf{x}_{\mathrm{i}}\right) \frac{\partial \mathrm{f}_{1}}{\partial \mathrm{y}}\left(\mathbf{x}_{\mathrm{i}}\right) \frac{\partial \mathrm{f}_{1}}{\partial \mathrm{z}}\left(\mathbf{x}_{\mathrm{i}}\right)\right]\left(\mathbf{R}^{-1}-\mathbf{I}\right)\left[\begin{array}{c}
0 \\
\mathrm{~b}\left(\mathbf{x}_{\mathrm{i}}\right) \\
0
\end{array}\right]
\end{aligned}
$$

for $\beta$. Extending this by minimizing the differences between $f_{1}\left(\mathbf{x}_{i}, \beta\right)$ and $f_{2}\left(\mathbf{x}_{i}, \beta\right)$ across all voxels we get

$$
\begin{aligned}
& {\left[\begin{array}{c}
f_{1}\left(\mathbf{x}_{1}\right)-f_{2}\left(\mathbf{x}_{1}\right) \\
f_{1}\left(\mathbf{x}_{2}\right)-f_{2}\left(\mathbf{x}_{2}\right) \\
\vdots \\
f_{1}\left(\mathbf{x}_{n}\right)-f_{2}\left(\mathbf{x}_{n}\right)
\end{array}\right]}
\end{aligned}
$$

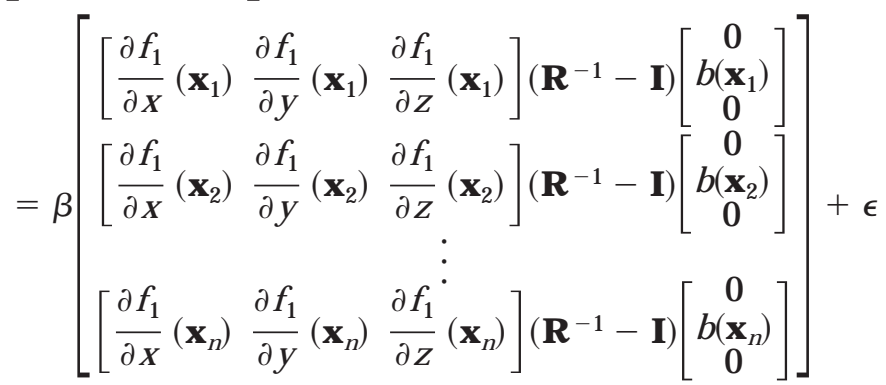

or in a more convenient notation

$$
\left[\mathbf{f}_{1}-\mathbf{f}_{2}\right]=\beta\left[\left(\left(\nabla \mathbf{f}_{1}\right) \mathbf{R}^{-1}-\nabla \mathbf{f}_{1}\right)_{\mathrm{y}} \times \mathrm{b}(\mathbf{X})\right]+\epsilon,
$$

where $\nabla$ denotes gradient, the $y$ subscript indicates that only that component is considered, $x$ denotes Hadamard (or direct) product and $b(\mathbf{X})$ denotes the column vector of deformations at all locations $\mathbf{x}_{1}$, $\mathbf{x}_{2} \cdots \mathbf{x}_{\mathrm{n}}$.

Of course we do not know the shape of the deformations, but we can model them as a linear combination of "known" basis-warps. In this paper we use the discrete cosine set of transformations (DCT), which has previously been used to represent inter-subject shape differences (Ashburner and Friston, 1999b). Equation 8 then changes to

$$
\begin{aligned}
& {\left[\mathbf{f}_{1}-\mathbf{f}_{2}\right]} \\
& \quad=\left\lfloor\mathbf{D}_{\mathrm{y}} \times \mathrm{b}_{1}(\mathbf{X}) \mathbf{D}_{\mathrm{y}} \times \mathrm{b}_{2}(\mathbf{X}) \cdots \mathbf{D}_{\mathrm{y}} \times \mathrm{b}_{1}(\mathbf{X})\right\rfloor \boldsymbol{\beta}+\epsilon,
\end{aligned}
$$

where $\mathbf{D}_{\mathrm{y}}$ denotes $\left(\left(\nabla \mathbf{f}_{1}\right) \mathbf{R}^{-1}-\nabla \mathbf{f}_{1}\right)_{\mathrm{y}}, \mathrm{b}_{1}$ to $\mathrm{b}_{1}$ denotes the set of I basis warps of increasing frequency and where $\boldsymbol{\beta}$ is an I $\times 1$ column vector of parameter estimates.

Finally, let us extend this to the entire time series, thereby restricting the solution to a single deformation field that is common to all time points. Let us denote, as above, the transformation matrix mapping $f_{i}$ onto $f_{1}$ by $\mathbf{T}_{i}$, and the corresponding rotation matrix with $\mathbf{R}_{i}$. Further let $\left(\left(\nabla \mathbf{f}_{1}\right) \mathbf{R}_{\mathrm{i}}^{-1}-\nabla \mathbf{f}_{1}\right)_{\mathrm{y}}$ be denoted by $\mathbf{D}_{\mathrm{iy}}$. Then a model for the time series becomes

$$
\begin{aligned}
& {\left[\begin{array}{c}
\mathbf{f}_{1}-\mathbf{f}_{2} \\
\mathbf{f}_{1}-\mathbf{f}_{3} \\
\vdots \\
\mathbf{f}_{1}-\mathbf{f}_{\mathrm{m}}
\end{array}\right]} \\
& \begin{aligned}
= & {\left[\begin{array}{cccc}
\mathbf{D}_{2 y} \times b_{1}(\mathbf{X}) & \mathbf{D}_{2 y} \times b_{2}(\mathbf{X}) & \cdots & \mathbf{D}_{2 y} \times b_{1}(\mathbf{X}) \\
\mathbf{D}_{3 y} \times b_{1}(\mathbf{X}) & \mathbf{D}_{3 y} \times b_{2}(\mathbf{X}) & \cdots & \mathbf{D}_{3 y} \times b_{1}(\mathbf{X}) \\
\vdots & \vdots & \ddots & \vdots \\
\mathbf{D}_{\text {my }} \times b_{1}(\mathbf{X}) & \mathbf{D}_{m y} \times b_{2}(\mathbf{X}) & \cdots & \mathbf{D}_{m y} \times b_{1}(\mathbf{X})
\end{array}\right] } \\
& \cdot \boldsymbol{\beta}+\epsilon
\end{aligned}
\end{aligned}
$$

or

$$
\mathbf{y}_{\mathrm{mn} \times 1}=\mathbf{A}_{\mathrm{mn} \times \mid} \boldsymbol{\beta}_{\mid \times 1}+\epsilon_{\mathrm{mn} \times 1},
$$

which is solved for $\boldsymbol{\beta}$ in a least squares sense through $\hat{\boldsymbol{\beta}}=\left(\mathbf{A}^{\top} \mathbf{A}\right)^{-1} \mathbf{A}^{\top} \mathbf{y}$. By iterative resampling of images and derivatives based on the current estimate of the deformation field, we can estimate the deformation field based on our forward model. The problem with the suggested model is that our design matrix $\mathbf{A}$ gets prohibitively large. For a typical study, $\mathrm{n}$ (number of voxels) is $64^{3}, \mathrm{~m}$ (number of scans) is in the order of hundreds (say 200), and I (the number of basis warps) is in the order $8^{3}$ to $12^{3}$. Hence a typical size of $\mathbf{A}$ would be $52 \cdot 10^{6} \times 1000$, and even though we need never store $\mathbf{A}$ explicitly, $\mathbf{A}^{\top} \mathbf{A}$ would consist of more than half a million unique elements, each requiring 52 million multiplications and additions for its estimation.

Luckily the form of $\mathbf{A}$ allows the same form of speed up as suggested by Ashburner and Friston (1999b). Briefly, it capitalizes on the factorization of the basis warps given by

$$
\left[b_{1}(\mathbf{X}) b_{2}(\mathbf{X}) \cdots b_{1}(\mathbf{X})\right]=\mathbf{B}=\mathbf{B}_{\mathrm{z}} \otimes \mathbf{B}_{\mathrm{y}} \otimes \mathbf{B}_{\mathrm{x}}
$$

where $\otimes$ denotes Kronecker tensor product and where $\mathbf{B}_{x}$ is the $n_{x} \times I_{x}$ matrix of the first $I_{x}$ components of a DCT defined on a grid of size $n_{x}$, and where $n_{x}$ is the size (in voxels) of the image volume in the x-direction, 

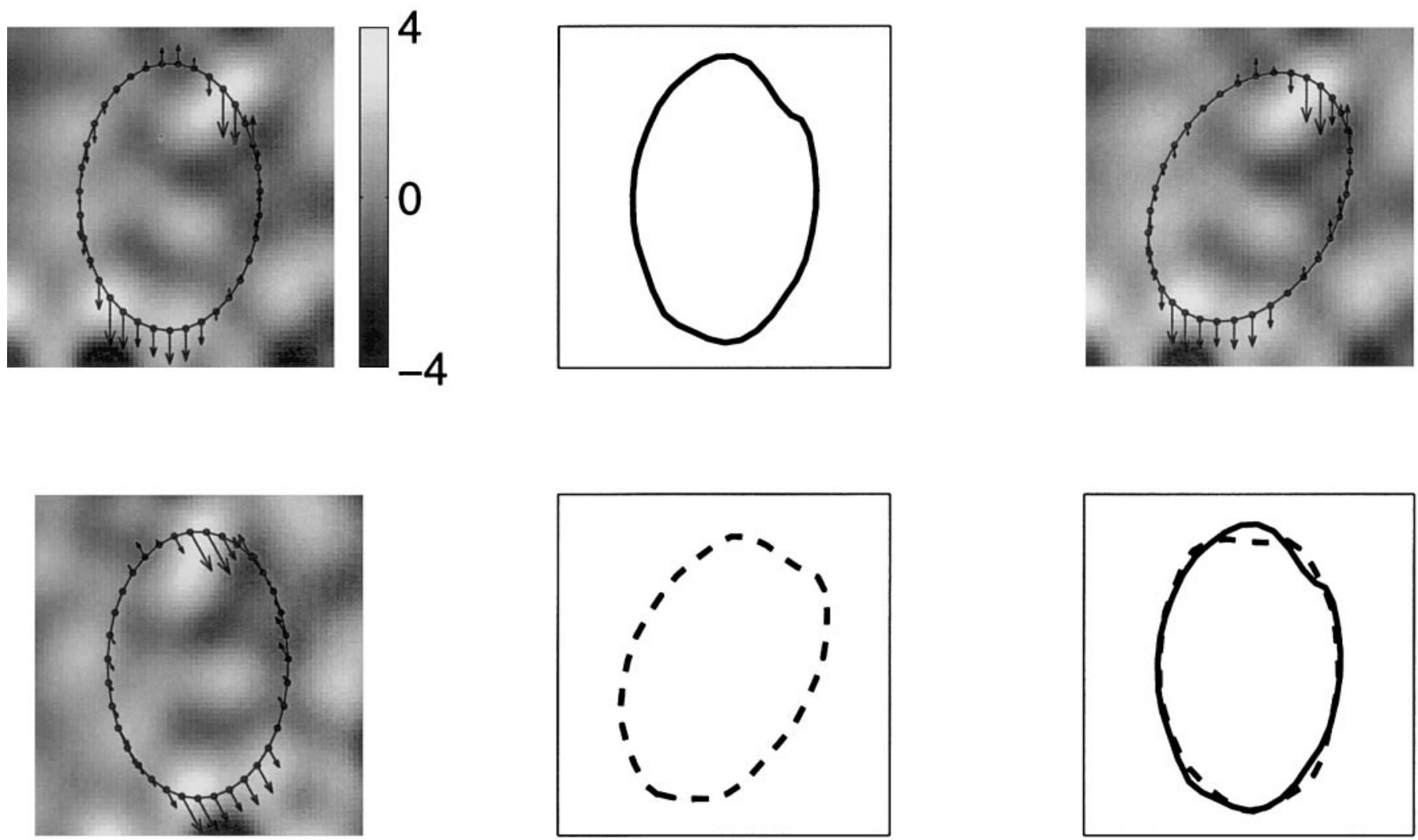

FIG. 2. Graphical explanation of the forward model defined by Eqs. (13) and (15). Upper left panel: shows an example of a random deformation field. The grey values indicate the deviation from a regular grid when sampling an object, where bright shades indicate sampling points have been deflected upwards and dark shades indicate downwards deflection. The color bar is graded in units of voxels. The contour indicates a vaguely brain like shape and the arrows shows direction and magnitude of apparent deformation of such an object when being imaged in this deformation field. Upper middle panel: shows the resulting deformed contour. Upper right panel: shows the object following a $30^{\circ}$ rotation, where the deformation field is stationary with respect to the scanner. N ote how each point on the contour has now moved to a different part of the deformation field, and is now associated with a different arrow compared to the unrotated case. Lower left panel: shows the same situation in a realigned (object) framework. Note how each point is affected by a deformation of different magnitude acting in a different direction compared to the unrotated case. Lower middle panel: shows the resulting deformed contour for the rotated case. Lower right panel: demonstrates the mismatch between two images of an identical object imaged in identical deformation fields, but where, in one case, the object had been rotated with respect to both the phase encode direction and the deformation field. N ote that according to this model differential deformations will be introduced by rotations and translations alike. Note also how for a given field and "movement" the mismatch is larger than that given by Eqs. (1) and (3) (Fig. 1), since there are now two effects contributing to it.

and where $\mathrm{I}_{\mathrm{x}}$ is the order of the warp in the $\mathrm{x}$-direction (equivalent for $\mathbf{B}_{\mathrm{y}}$ and $\mathbf{B}_{z}$ ).

\section{Model for I nhomogeneities Fixed with Respect to Scanner (Mode 2)}

As our next model consider the case where the deformation field is fixed with respect to the scanner frame, e.g., as a result of a poor/failed shim. Equation (1) above, describing the forward model, becomes

$$
\mathrm{f}_{1}(\mathbf{X}+\mathbf{d}(\mathbf{X}))
$$

for the stationary image and

$$
\mathrm{f}_{2}\left(\mathbf{T}^{-1} \mathbf{X}+\mathbf{R}^{-1} \mathbf{d}(\mathbf{X})\right)
$$

for the moved image which, after realignment, means that we equate $f_{1}(\mathbf{X}+\mathbf{d}(\mathbf{X}))$ with

$$
\mathrm{f}_{2}\left(\mathbf{T T}^{-1} \mathbf{X}+\mathbf{R}^{-1} \mathbf{d}(\mathbf{T X})\right)=\mathrm{f}_{2}\left(\mathbf{X}+\mathbf{R}^{-1} \mathbf{d}(\mathbf{T X})\right) .
$$

The intuition for this model is to consider a distorting mirror at a fair ground. Clearly the deformation field is stationary in time, but as you move your head in front of the mirror the image you "acquire" will change since you sample the deformation field at different positions. Even if you were able to "realign" these images they would still look different due to differential deformations. Figure 2 illustrates this model.

Making the same assumption as above regarding the "known" shape of the deformation field and the exis- 
tence of an inverse field, the image intensities of the stationary and moved scans depend on $\beta$ according to

$$
\begin{aligned}
& \mathrm{f}_{1}\left(\mathbf{x}_{\mathrm{i}}, \beta\right) \approx \mathrm{f}_{1}\left(\mathbf{x}_{\mathrm{i}}, 0\right) \\
& \quad+\beta\left[\frac{\partial \mathrm{f}_{1}}{\partial \mathrm{x}}\left(\mathbf{x}_{\mathrm{i}}\right) \frac{\partial \mathrm{f}_{1}}{\partial \mathrm{y}}\left(\mathbf{x}_{\mathrm{i}}\right) \frac{\partial \mathrm{f}_{1}}{\partial \mathrm{z}}\left(\mathbf{x}_{\mathrm{i}}\right)\right]\left[\begin{array}{c}
0 \\
\mathrm{~b}\left(\mathbf{x}_{\mathrm{i}}\right) \\
0
\end{array}\right]
\end{aligned}
$$

and

$$
\begin{aligned}
& \mathrm{f}_{2}\left(\mathbf{x}_{\mathrm{i}}, \beta\right) \approx \mathrm{f}_{2}\left(\mathbf{x}_{\mathrm{i}}, 0\right) \\
& \quad+\beta\left[\frac{\partial \mathrm{f}_{1}}{\partial \mathrm{x}}\left(\mathbf{x}_{\mathrm{i}}\right) \frac{\partial \mathrm{f}_{1}}{\partial \mathrm{y}}\left(\mathbf{x}_{\mathrm{i}}\right) \frac{\partial \mathrm{f}_{1}}{\partial \mathrm{z}}\left(\mathbf{x}_{\mathrm{i}}\right)\right] \mathbf{R}^{-1}\left[\mathrm{~b}\left(\begin{array}{c}
0 \\
\left.\mathbf{T}\left[\begin{array}{c}
\mathbf{x}_{\mathrm{i}} \\
1 \\
0
\end{array}\right]\right)
\end{array}\right]\right.
\end{aligned}
$$

Extending this to all voxels gives us the equivalent to Eq. (8)

$$
\begin{aligned}
{\left[\mathbf{f}_{1}-\mathbf{f}_{2}\right]=\beta\left[\left(\left(\nabla \mathbf{f}_{1}\right) \mathbf{R}^{-1}\right)_{\mathrm{y}} \times \mathrm{b}(\right.} & \left.\left(\mathbf{T}\left[\begin{array}{c}
\mathbf{X}^{\top} \\
\mathbf{1}_{1 \times \mathrm{n}}
\end{array}\right]\right)^{\top}\right) \\
& \left.-\nabla \mathbf{f}_{1 \mathrm{y}} \times \mathrm{b}(\mathbf{X})\right]+\epsilon .
\end{aligned}
$$

Recognizing that for a field modeled as a linear combination of basis warps $\mathrm{b}(\mathbf{T X})=\beta_{1} \mathrm{~b}_{1}(\mathbf{T X})+$ $\beta_{2} b_{2}(\mathbf{T X})+\ldots \beta_{1} b_{1}(\mathbf{T X})$ (where TX is used as a shorthand for the one-extended formulation) we get

$$
\begin{aligned}
{\left[\mathbf{f}_{1}-\right.} & \left.\mathbf{f}_{2}\right] \\
= & {\left[\left(\mathbf{D R}^{-1}\right)_{\mathrm{y}} \times \mathrm{b}_{1}(\mathbf{T X})-\mathbf{D}_{\mathrm{y}} \times \mathrm{b}_{1}(\mathbf{X}) \cdots\left(\mathbf{D R}^{-1}\right)_{\mathrm{y}}\right.} \\
& \left.\times \mathrm{b}_{\mathrm{l}}(\mathbf{T X})-\mathbf{D}_{\mathrm{y}} \times \mathrm{b}_{\mathrm{l}}(\mathbf{X})\right] \boldsymbol{\beta}+\epsilon .
\end{aligned}
$$

Extending it to the entire time series gives

$$
\left[\begin{array}{c}
\mathbf{f}_{1}-\mathbf{f}_{2} \\
\mathbf{f}_{1}-\mathbf{f}_{3} \\
\vdots \\
\mathbf{f}_{1}-\mathbf{f}_{\mathrm{m}}
\end{array}\right]=\left[\begin{array}{c}
\left(\mathbf{D R} \mathbf{R}_{2}^{-1}\right)_{\mathrm{y}} \times \mathrm{b}_{1}\left(\mathbf{T}_{2} \mathbf{X}\right)-\mathbf{D}_{\mathrm{y}} \times \mathrm{b}_{1}(\mathbf{X}) \cdots(\mathbf{D R}-1)_{\mathrm{y}} \times \mathrm{b}_{1}\left(\mathbf{T}_{2} \mathbf{X}\right)-\mathbf{D}_{\mathrm{y}} \times \mathrm{b}_{\mathrm{l}}(\mathbf{X}) \\
\left(\mathbf{D R} \mathbf{R}^{-1}\right)_{\mathrm{y}} \times \mathrm{b}_{1}\left(\mathbf{T}_{3} \mathbf{X}\right)-\mathbf{D}_{\mathrm{y}} \times \mathrm{b}_{1}(\mathbf{X}) \cdots\left(\mathbf{D R} \mathbf{R}_{3}^{-1}\right)_{\mathrm{y}} \times \mathrm{b}_{1}\left(\mathbf{T}_{3} \mathbf{X}\right)-\mathbf{D}_{\mathrm{y}} \times \mathrm{b}_{\mathrm{l}}(\mathbf{X}) \\
\vdots \\
\vdots \\
\left(\mathbf{D R} \mathbf{R}_{\mathrm{m}}^{-1}\right)_{\mathrm{y}} \times \mathrm{b}_{1}\left(\mathbf{T}_{\mathrm{m}} \mathbf{X}\right)-\mathbf{D}_{\mathrm{y}} \times \mathrm{b}_{1}(\mathbf{X}) \cdots\left(\mathbf{D R} \mathbf{R}_{\mathrm{m}}^{-1}\right)_{\mathrm{y}} \times \mathrm{b}_{\mathrm{l}}\left(\mathbf{T}_{\mathrm{m}} \mathbf{X}\right)-\mathbf{D}_{\mathrm{y}} \times \mathrm{b}_{\mathrm{l}}(\mathbf{X})
\end{array}\right] \boldsymbol{\beta}+\epsilon
$$

which may again be solved iteratively in a least squares sense.

Model for Inhomogeneities Changing with the Position of the Object (Model 3)

The final model is where the distorting field changes as the subject moves in the scanner. An example of this would be where the head, a convex object with aircavities, is subject to out of plane rotation such that new configurations of air to matter are encountered by the magnetic flux. Because the resulting magnetization changes as the subject moves, there is no single deformation field common to all the images and the problem may seem intractable. However, we can reformulate the problem and attempt to model the derivatives of the deformation field with respect to object movement. Suppose we have a field $d(\mathbf{x}, \mathbf{q})$, which describes the deformation at a position $\mathbf{x}$ in the space of an object positioned according to some parameter vector $\mathbf{q}$. Then the deformation for a position $\mathbf{q}+\Delta \mathbf{q}$ can be approximated with the first two terms of a Taylor expansion as

$$
\begin{aligned}
\mathrm{d}(\mathbf{x}, \mathbf{q} & +\Delta \mathbf{q}) \approx \mathrm{d}(\mathbf{x}, \mathbf{q}) \\
& +\left[\frac{\partial \mathrm{d}}{\partial \mathrm{q}_{1}}(\mathbf{x}, \mathbf{q}) \frac{\partial \mathrm{d}}{\partial q_{2}}(\mathbf{x}, \mathbf{q}) \cdots \frac{\partial \mathrm{d}}{\partial \mathrm{q}_{\mathrm{k}}}(\mathbf{x}, \mathbf{q})\right] \Delta \mathbf{q}
\end{aligned}
$$

where $\mathrm{k}$ is the number of parameters needed to de scribe the position. Now the actual positions in which we sample our stationary and moved images become the arguments of

$$
\mathrm{f}_{1}(\mathbf{X}+\mathbf{d}(\mathbf{X}))
$$

and

$$
\mathrm{f}_{2}\left(\mathbf{T}^{-1} \mathbf{X}+\mathbf{R}^{-1} \mathbf{d}(\mathbf{X})+\mathbf{R}^{-1} \mathrm{D}(\mathbf{d}(\mathbf{X})) \Delta \mathbf{q}\right),
$$

respectively, where $D(\mathbf{d}(\mathbf{X}))$ denotes the partial derivatives of $\mathbf{d}_{\mathrm{y}}$ with respect to $\mathbf{q}$ evaluated at all points $\mathbf{X}$. We will derive a model where we attempt to estimate both the field at the point in position space given by $\mathbf{q}$, and its partial derivatives with respect to q. This implies that we simultaneously model $\mathrm{k}+1$ fields, which remains tractable providing that $m \gg k$.

We assume again that the fields are known (denoted $\left.b, \partial b / \partial q_{1}, \partial b / \partial q_{2}, \ldots, \partial b / \partial q_{k}\right)$ and that the corresponding scalars are given by $\beta, \beta^{1}, \beta^{2}, \ldots, \beta^{k}$. Then the dependence of our stationary image on $\beta-\beta^{k}$ becomes

$$
\mathrm{f}_{1}\left(\mathbf{x}_{\mathrm{i}}, \beta\right) \approx \mathrm{f}_{1}\left(\mathbf{x}_{\mathrm{i}}\right)+\beta\left[\frac{\partial \mathrm{f}_{1}}{\partial \mathrm{x}}\left(\mathbf{x}_{\mathrm{i}}\right) \frac{\partial \mathrm{f}_{1}}{\partial \mathrm{y}}\left(\mathbf{x}_{\mathrm{i}}\right) \frac{\partial \mathrm{f}_{1}}{\partial \mathrm{z}}\left(\mathbf{x}_{\mathrm{i}}\right)\right]\left[\begin{array}{c}
0 \\
\mathrm{~b}\left(\mathbf{x}_{\mathrm{i}}\right) \\
0
\end{array}\right]
$$


and for the moved image

$$
\begin{aligned}
\mathrm{f}_{2}\left(\mathbf{x}_{\mathrm{i}}, \beta\right) \approx \mathrm{f}_{2}\left(\mathbf{x}_{\mathrm{i}}\right)+\beta\left(\nabla \mathrm{f}_{1}\left(\mathbf{x}_{\mathrm{i}}\right)\right) \mathbf{R}^{-1}\left[\begin{array}{c}
0 \\
\mathrm{~b}\left(\mathbf{x}_{\mathrm{i}}\right) \\
0
\end{array}\right] \\
+\beta^{1}\left(\nabla \mathrm{f}_{1}\left(\mathbf{x}_{\mathrm{i}}\right)\right) \mathbf{R}^{-1}\left[\begin{array}{c}
0 \\
\frac{\partial \mathrm{b}}{\partial \mathrm{q}_{1}}\left(\mathbf{x}_{\mathrm{i}}\right) \\
0
\end{array}\right] \Delta \mathrm{q}_{1}+\cdots
\end{aligned}
$$

By analogy with the earlier models we can solve for $\beta-\beta^{k}$ in a least squares sense through

$$
\begin{array}{r}
{\left[\mathbf{f}_{1}-\mathbf{f}_{2}\right]=\left[\left(\left(\nabla \mathbf{f}_{1}\right) \mathbf{R}^{-1}-\nabla \mathbf{f}_{1}\right)_{\mathrm{y}} \times \mathrm{b}(\mathbf{X}) \Delta \mathrm{q}_{1}\left(\Delta \mathbf{f}_{1}\right) \mathbf{R}^{-1}\right.} \\
\left.\times \frac{\partial \mathrm{b}}{\partial \mathrm{q}_{1}}(\mathbf{X}) \cdots\right]\left[\begin{array}{c}
\beta \\
\beta^{1} \\
\vdots
\end{array}\right]+\epsilon .
\end{array}
$$

If we model both the field at object position $\mathbf{q}$ and itsderivatives as linear combinations of DCT basis warps we obtain

$$
\begin{aligned}
{\left[\mathbf{f}_{1}-\mathbf{f}_{2}\right] } & \\
= & {\left[\left(\mathbf{D} \mathbf{R}^{-1}-\mathbf{D}\right) \times \mathbf{b}_{1} \cdots\left(\mathbf{D R}^{-1}-\mathbf{D}\right) \times \mathbf{b}_{1}\right.} \\
& \Delta \mathrm{q}_{1} \mathbf{D} \mathbf{R}^{-1} \times \mathbf{b}_{1} \Delta \mathrm{q}_{1} \mathbf{D R}^{-1} \times \mathbf{b}_{2} \cdots \\
& \left.\Delta \mathrm{q}_{\mathrm{k}} \mathbf{D R}^{-1} \times \mathbf{b}_{1}\right] \times\left[\begin{array}{c}
\boldsymbol{\beta} \\
\boldsymbol{\beta}^{1} \\
\vdots \\
\boldsymbol{\beta}^{\mathrm{k}}
\end{array}\right]+\epsilon
\end{aligned}
$$

where $\mathbf{D}$ denotes $\nabla \mathbf{f}_{1}, \mathbf{D} \mathbf{R}^{-1}$ denotes $\left(\nabla \mathbf{f}_{1}\right) \mathbf{R}^{-1}, \mathbf{b}_{\mathrm{i}}$ denotes the ith basis warps evaluated at all points in $\mathbf{X}$, and where it is implicit that only the y-direction is considered. Note that all fields are now modeled using the same basis set, but each field is still distinct because it comprises different linear combinations as given by the $\mathrm{k}+1$ column vectors $\boldsymbol{\beta}-\boldsymbol{\beta}^{\mathrm{k}}$ of size I $\times 1$.

Finally we extend the model to the full time series

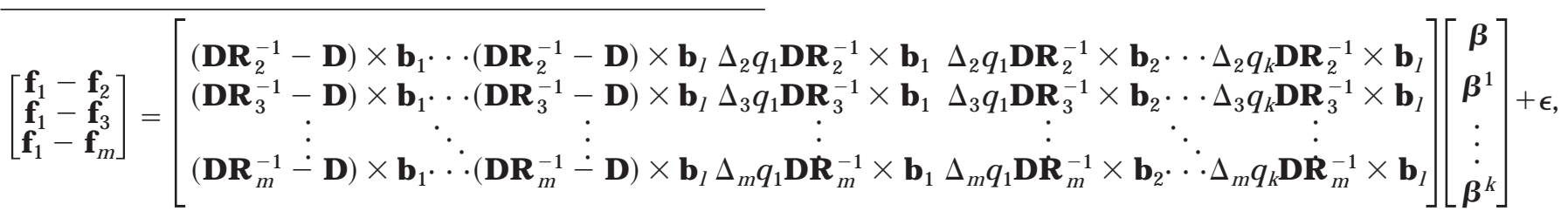

where $\Delta_{i} q_{j}$ denotes the difference in object position parameter $\mathrm{j}$ between the ith scan and scan 1.

The two previous models can both be seen as special cases of this "general" model, in that Eq. (28) reduces to the first model (Eq. (10)) when $\boldsymbol{\beta}^{1}-\boldsymbol{\beta}^{\mathrm{k}}$ are all 0. Furthermore, when the "true" model conforms to Eq. (20), the general model, using the three transIations and three rotations as $\mathbf{q}$, gives a good approximation. In this instance $\boldsymbol{\beta}^{1}-\boldsymbol{\beta}^{\mathrm{k}}$ would generate derivative fields that are simply the partial derivatives of the stationary field with respect to translations and rotations of the field itself. It may seem unwarranted to formulate a model that has a design matrix the size of $\mathrm{mn} \times(\mathrm{k}+1) \mathrm{l}$, corresponding to about 52 . $10^{6} \times 7000$ (assuming $20064 \times 64 \times 64$ scans modeling fields with $10 \times 10 \times 10$ basis functions) if we want only to model a static field. However, the form of the design matrix in Eq. (28) lends itself to the Kronecker product formulation, making the inversion of $\mathbf{A}^{\top} \mathbf{A}$ (of size $\left.(k+1)|\times(k+1)|\right)$ the rate limiting step, rather than its calculation.

One may often be interested in letting $\mathbf{q}$ be a subset of the parameters describing the rigid body transformation.
For example, we could postulate that, for a head moving in a well-shimmed scanner, the main source of deformations is induced by the head itself. These deformations would follow the head (i.e., move according to model 1), but, in addition, change in the presence of rotations around the $x$ - and/or $y$-axis. One would model this case using Eq. (28) with q containing only those rotation angles (needless to say all rotation parameters are used for $\mathbf{R}_{\mathrm{i}}$ ), yielding a design matrix of size $m n \times 3$ l.

Empirical studies may show that the effect of the differences in the direction of the deformation field (i.e., model 1, or the first I col umns of Eq. (28)) is small compared to the effects of the actual changes of the deformation field. If that is the case we can drop the I first columns of Eq. (28). It should be noted that in this case we would only model the derivatives of the deformations and would be effectively "unwarping" all images to a common deformation state given by the first image volume in the series (as opposed to some "zero deformation" state). This models the deformation-by-movement interactions, but not the deformations per se.

Finally we note that one is not restricted to modeling the deformation field as a first order Taylor expansion. 
In the presence of large movement a second term can be added to Eq. (21)

$$
\begin{aligned}
& \mathrm{d}(\mathbf{q}+\Delta \mathbf{q}) \approx \mathrm{d}(\mathbf{q})+\left[\frac{\partial \mathrm{d}}{\partial \mathrm{q}_{1}} \frac{\partial \mathrm{d}}{\partial \mathrm{q}_{2}} \cdots \frac{\partial \mathrm{d}}{\partial \mathrm{q}_{\mathrm{k}}}\right] \Delta \mathbf{q} \\
& +\Delta \mathbf{q}^{\top}\left[\begin{array}{cccc}
\frac{\partial^{2} d}{\partial q_{1}^{2}} & \frac{\partial^{2} d}{\partial q_{1} \partial q_{2}} & \cdots & \frac{\partial^{2} d}{\partial q_{1} \partial q_{k}} \\
\frac{\partial^{2} d}{\partial q_{1} \partial q_{2}} & \frac{\partial^{2} d}{\partial q_{2}^{2}} & \cdots & \frac{\partial^{2} d}{\partial q_{2} \partial q_{k}} \\
\vdots & \vdots & \ddots & \vdots \\
\frac{\partial^{2} d}{\partial q_{1} \partial q_{k}} & \frac{\partial^{2} d}{\partial q_{2} \partial q_{k}} & \cdots & \frac{\partial^{2} d}{\partial q_{k}^{2}}
\end{array}\right] \Delta \mathbf{q}
\end{aligned}
$$

Modeling $1+\mathrm{k}+\left(\begin{array}{c}\mathrm{k}+1 \\ 2\end{array}\right)$ fiel ds. It is straightforward to extend Eq. (28) in this case. This model may seem extravagant, but considering the special case above, where we concentrate on the effects of out-of-plane rotations, one would only model 5 fields, which is perfectly feasible with current computing power.

\section{METHODS}

\section{Simulated Data}

A $64 \times 64 \times 64$ EPI image volume with isotropic $3-\mathrm{mm}$ voxels was acquired on a 2 Tesla Magnetom VISION (Siemens, Erlangen) MRI scanner. The image was one in a series constituting an epoch-related auditory fMRI study. Scan to scan repetition time was $7 \mathrm{~s}$ and TE $40 \mathrm{~ms}$. A slice $40 \mathrm{~mm}$ above the AC-PC plane was selected for the 2-D simulations.

Translations and rotations for time points 2 to 25 were created such that $p_{i}(j+1)=p_{i}(j)+N(0,1)$ (in units of $\mathrm{mm}$ and degrees) for the two translations and the rotation relevant for the $2-D$ case. Random $8 \times 8$ deformation fiel ds were created according to $\left(\mathbf{B}_{\mathrm{y}} \otimes \mathbf{B}_{\mathrm{x}}\right) \mathbf{b}$, where $\mathbf{b}$ is a random independent identically distributed (iid) $\mathrm{N}(0,5)$ $64 \times 1$ column vector. Clearly these random fields have a spatial structure quite different from those observed in actual field maps, but the magnitude of distortions is comparable, ranging from -2 to 2 pixels.

From the single 2-D image described above, time series of variable length were created based on the random movements and deformation fields. Time series were created according to both model 1 (field moving with subject) and model 2 (field stationary in scanner) using Eqs. (3) and (15), respectively. Varying levels of iid Gaussian noise was added to the images.

Singular value decomposition (SVD) of voxel mean corrected time series was used as a device to assess the spatiotemporal modes of variance before and after correction for deformations.
Data created according to the forward model 1 (Eq. (3)) was analyzed according to the corresponding estimation model (Eq. (10)) and data created according to forward model 2 (Eq. (15)) was analyzed with Eq. (20). In addition, data created according to forward models 1 and 2 were analyzed with the general estimation model given by $\mathrm{Eq}$. (28).

\section{Empirical Data}

A healthy male volunteer was positioned in a 2 Tesla Magnetom VISION (Siemens, Erlangen) MRI scanner. Scan to scan repetition time was $4.86 \mathrm{~s}$ with a TE of 40 $\mathrm{ms}$, and a total of $2964 \times 64 \div 64$ voxel EPI images with an isotropic voxel size of $3 \mathrm{~mm}$ were collected. The first four scans were discarded, leaving a total of 25 . Head movement was lightly constrained, and the subject was instructed to perform a head rotation around the $x$-axis (pitch) and/or the y-axis (roll) every five scans.

Movement parameters were estimated using SPM99 (Ashburner and Friston, 1999a) and used to model the derivatives of the deformation field with respect to pitch and roll using Eq. (28). SVD was used to characterize the variance within the time series before and after resampling based on the estimated deformation fields. Note that because we did not model a static field, this "undeformation" will be relative to the deformation extent at the geometric mean position of the time series.

\section{RESULTS}

\section{Simulated Data}

The simulations indicated that deformation fields were readily estimated when analyzing a time-series using an estimation model corresponding to the forward model that was used to generate the data. Examples of the results of a simulation are shown in Figs. 3 to 9. F or the simulations presented in Figs. 3 to 5 and 7 to 9, we used the deformation field shown in Figs. 1 and 2, scaled such that the deformations ranged from -2 to 2 voxels. F or the simulations presented in Fig. 6 another field, drawn from the same iid random vector b, was used. The time series was 25 scans in length, and the simulated movements are shown in Fig. 3. An SVD of the time series indicates that variance was dominated by movement and the eigenimages before "undeformation" exhibited a pattern determined by local magnitude of deformation and image gradient. This is consistent with observations based on real data. The deformation fields were estimated accurately when using estimation models corresponding to those used to generate the data, as evident from Figs. 4 and 5 . When "undeforming" the time series 90\% of the movementrelated variance was removed. This is reflected in the eigenimages before and after "undeformation" shown in Figs. 4 and 5. 

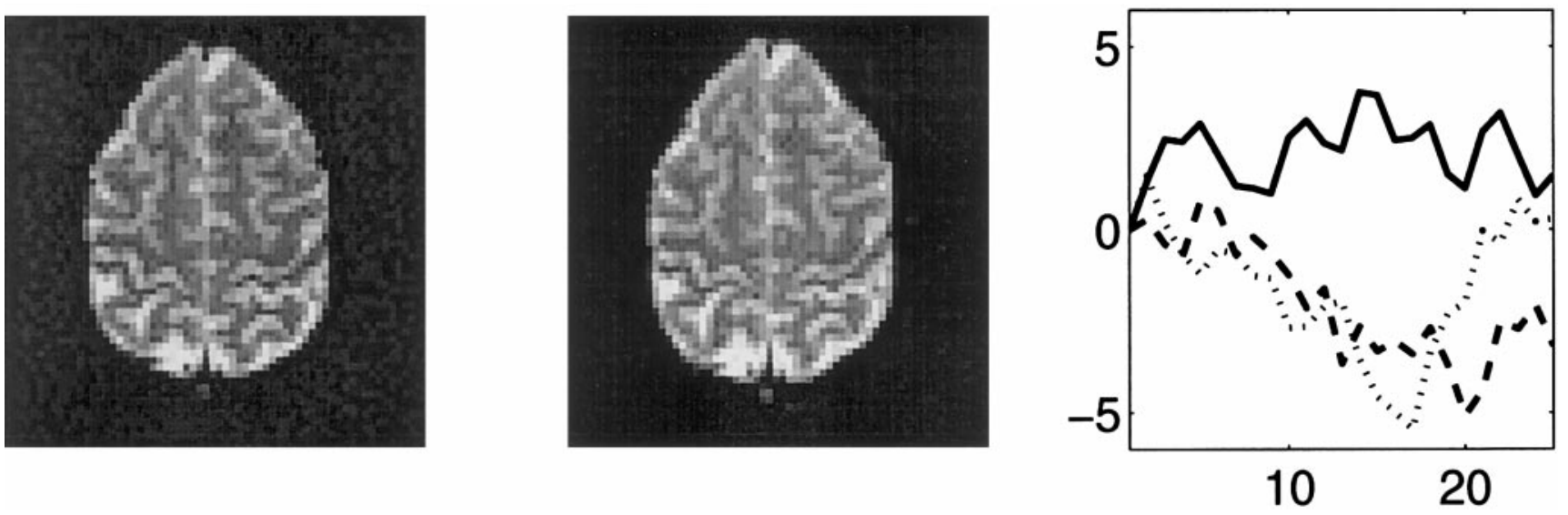

FIG. 3. Data used for simulations. Left panel: 2-D image used for simulations. Middle panel: Deformed image after having been imaged at the shown position in the deformation field that was used for the simulations. The deformation field shown in Figs. 1 and 2 was scaled to yield deformations of half the magnitude shown there (i.e., ranging from -2 to 2 voxels) and was used for all the simulations. Right panel: Translations (left-right, solid line; anterior-posterior, dashed line) and rotations (dotted line) in mm and degrees, respectively, that was used for all simulations.

For clarity we show results from a simulation where no white noise was added to the time series. However, the method appears stable in the presence of white noise. Simulations with added white noise to yield signal-to-noise ratios (SNR) ranging from 200 to 2 indicate that only when SNR drops to about 10 does accuracy go down appreciably (Fig. 6). Simulated timeseries as short as 10 scans still allowed accurate estimation. The movements in the simulation depicted in the figures are large compared to those typically observed. Simulations were performed for a large range of movements with similar results.

The general estimation model defined by Eq. (28) yields sensible results when applied to data generated by either models 1 or 2 (Eqs. (3) or (15)). When analyzing data from model 1 the estimation of the field at $\mathbf{q}_{0}$ (the first I columns in Eq. (28)) corresponds to the true field and the estimates of the derivative fields are very close to zero (Fig. 7). Because model 1 is a subset of the general model, the "undeformation" removed as much movement-related variance as the corresponding generative model and the first eigenimage was very similar to that in the lower right panel of Fig. 4 (data not shown). When analyzing data generated according to model 2, we estimated a static deformation field and derivatives with respect to three movement parameters (Fig. 8). For this case the "true" derivative fields were obtained from a numeric differentiation of the static deformation field itself. It can be seen that there are some differences between the "true" and the estimated fields. Furthermore, a location with large differences between the expected and estimated fields for one component tend to have large differences for one or more other components (e.g., at the anteriormost aspect of the brain). This is due to the correlation be- tween the movement parameters in this particular simulation (with, e.g., a -0.48 correlation between translation in the left-right direction and rotation), which tend to complicate the interpretation of the component fields. However, results, in terms of variance reduction, from the "undeformation" remain unchanged. The variance reduction is demonstrated in Fig. 9, which shows the first five eigenvalues for the "deformed" time series and for the time series "undeformed" according to model 2 and the general model. It is clear that a nearly equivalent variance reduction is achieved with the general model. It is not surprising that it is not identical because we employ a first order Taylor expansion in the general model. When including second order terms (i.e., according to Eq. (29)) this difference is virtually obliterated (data not shown).

\section{Empirical Data}

The analysis of the 25 scans of empirical data proceeded for five iterations, taking $\sim 30 \mathrm{~min}$ on a Sun Ultra 60 when modeling two derivative fields with $8 \times$ $8 \times 8$ basis functions. The resulting derivative fields looked reasonable (Fig. 10, a formal comparison to measured field maps will be reported elsewhere) and indicated large changes of deformations, with respect to movement, in known problematic areas such as around the sinuses and at the temporal poles. The rate of change of deformation, in the most affected areas, was in the order of 0.1 to 0.15 voxels per degree rotation of the head. Because areas with large susceptibility gradients tend to coincide with areas with large T2* gradients, this means that one degree of rotation may cause local intensity changes in the order of $5 \%$ (assuming a T2* image intensity gradient of $50 \%$ ). It is 

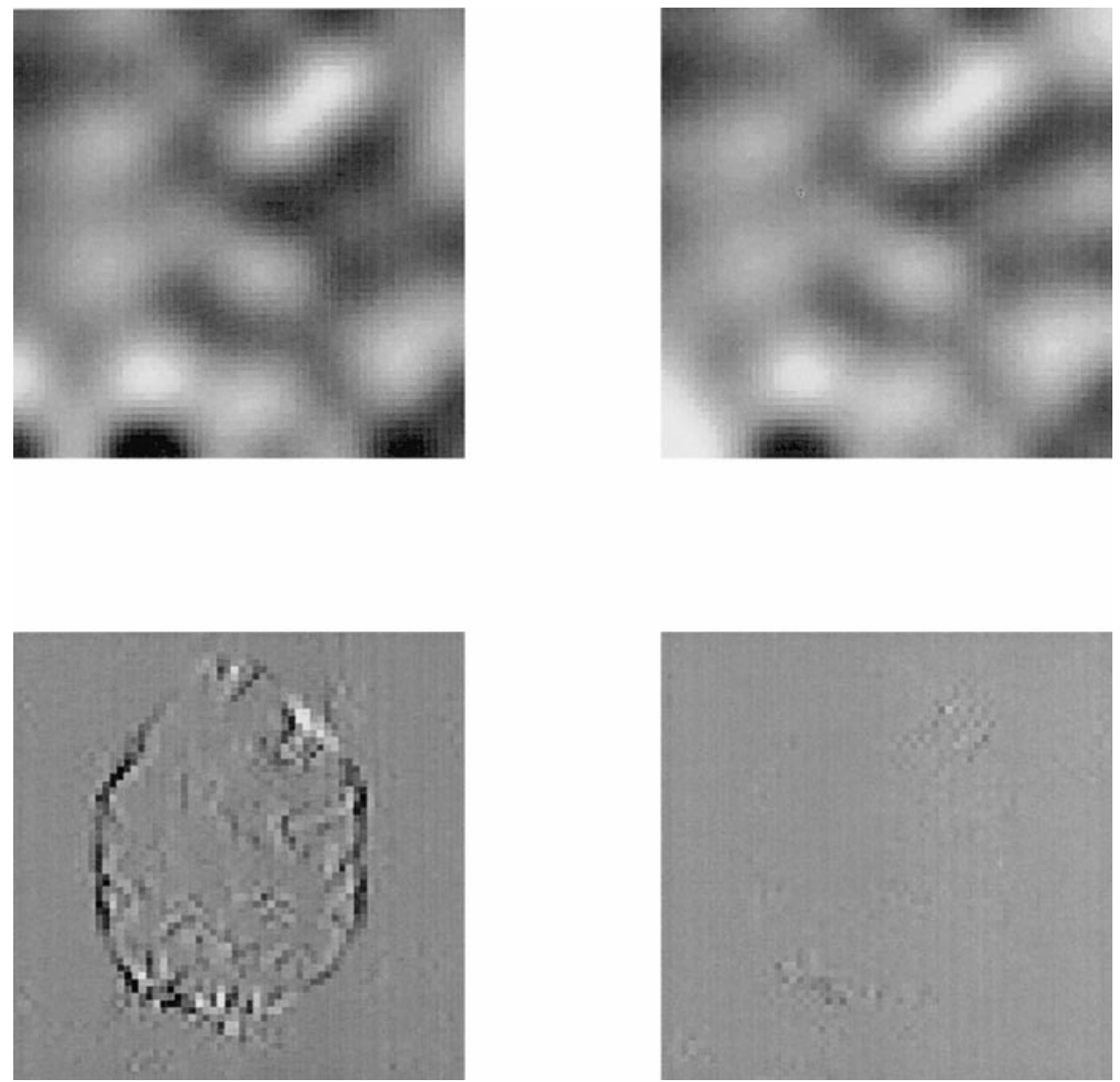

FIG. 4. Results from simulations generating data according to Eq. (3) and analyzing them using Eq. (10), i.e., using the model where the deformations are assumed to follow the object perfectly. The upper row shows the "true" and the estimated deformations fields, respectively, to the left and right. The lower left panel shows the first eigenimage (multiplied with the first singular value) of the SVD of the time series with the deformations in it. Note how the areas with high loadings correspond to those where we had large differences in Fig. 1 using the same deformation field. It should also be noted that the eigenvector corresponding to the first eigenimage has a $>0.99$ correlation with the rotations shown in Fig. 3. The lower right panel shows the same after "undeformation" based on the estimated deformation field.

interesting to note the structured appearance of these maps with a clearly discernible left-right symmetry for $\mathrm{x}$-axis rotations and corresponding antisymmetry for $y$-axis rotations. We stress that this is not a property that is conditional on our choice of basis-functions, which can model any field up to the specified cut-off frequency. Note also the pattern of opposite changes surrounding the air cavity in the sinuses (best visible in the transverse slices) showing the dipolar field introduced by this cavity.

The first eigenvector is clearly dominated by movement effects, being very close to a linear combination of the estimated rotation angles ( $F$ ig. 11 , middle and right panels). The second eigenvector is probably due to intrascan movement showing a strong correlation to the derivative of rotation around the y-axis. The eigenvector spectra before and after "undeformation" shows that variance has been removed from the time series, albeit less so than for the simulated data. The realignment alone removed $84.6 \%$ of the variance from this data set, and the "undeformation" removed an additional $4.7 \%$. Note though that this corresponds to $30 \%$ of the residual variance, and an even larger proportion of residual movement related variance. The spectra appear to indicate that a similar proportion of variance was removed from both the first and the second component (which was not directly related to the movement parameters). This is not the case. In fact the first and second eigenvectors have traded places before and after "undeformation." Since so much of the variance relating to the first eigenvector ( $\sim 49 \%)$ was re moved, it was relegated to second place to be preceded by the eigenvector relating to intrascan movement which changed very little ( $\sim 5 \%)$.

The eigenimage of the realigned time series is shown in Fig. 12 along with the corresponding scaled eigenimage of the "undeformed" time series. Appreciable amounts of movement-related variance have been re moved from the data, in particular from areas known to be influenced by susceptibility artefacts such as the 

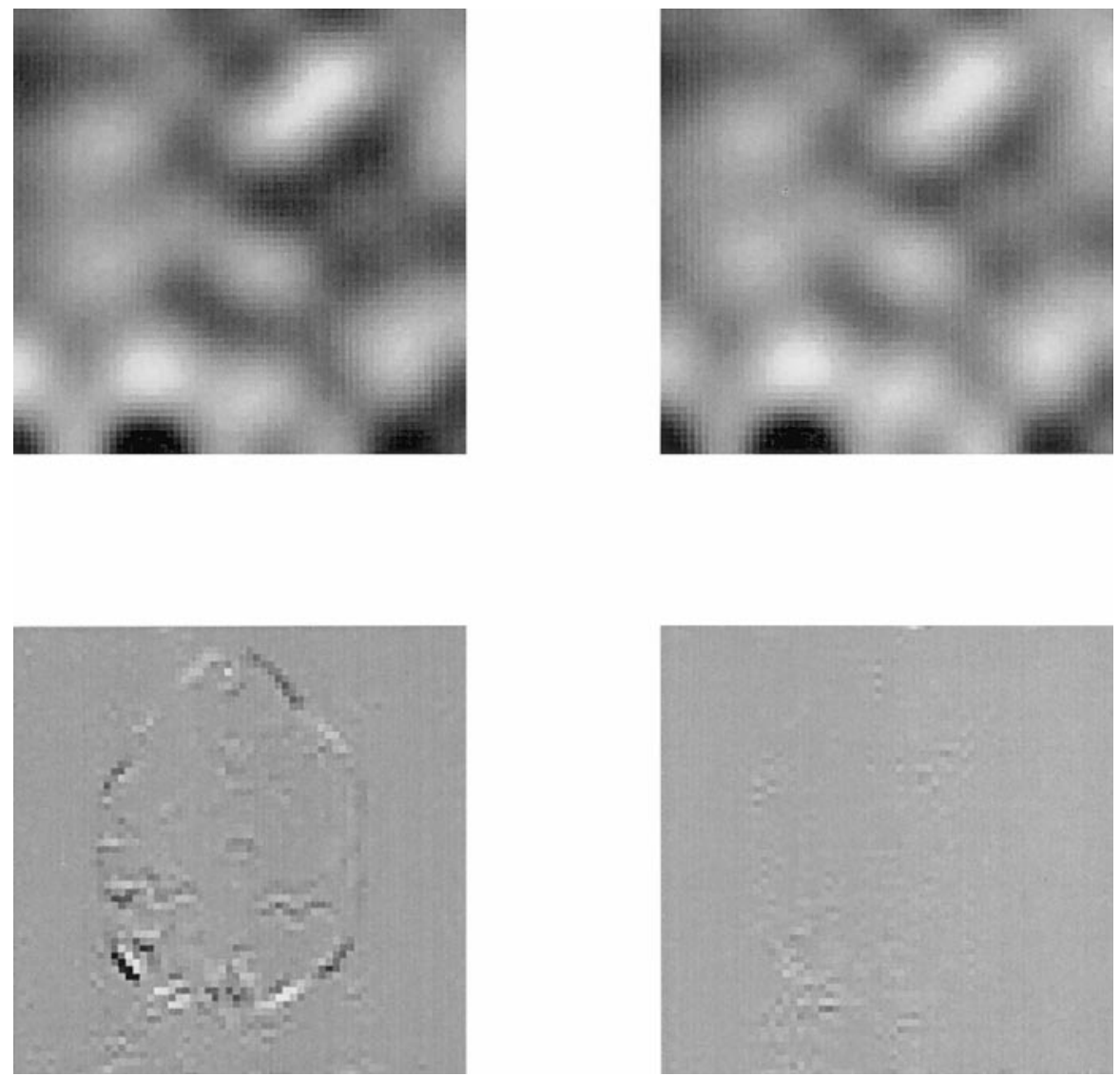

FIG. 5. Results from simulations generating data according to Eq. (15) and analyzing them using Eq. (20), i.e., using the model where the deformations are assumed to be stationary in the scanner framework. The upper row shows the "true" and the estimated deformations fields, respectively, to the left and right. The lower left panel shows the first eigenimage (multiplied with the first singular value) of the SVD of the time series with the deformations in it. Note how the areas with high loadings correspond to those where we had large differences in Fig. 1 using the same deformation field. It should also be noted that the eigenvector corresponding to the first eigenimage has a $>0.96$ correlation with the rotations shown in Fig. 3. The lower right panel shows the same after "undeformation" based on the estimated deformation field.

frontal cortex (seen best in the sagittal section) and the temporal poles (seen best in the coronal section).

\section{DISCUSSION}

It is evident that even after rigid-body registration, considerable movement-related variance is still present in the fMRI time series. There are a number of conceivable explanations for this, including (i) interpolation errors (Ostuni et al., 1997; Grootoonk et al., 2000), (ii) spin-history effects (Friston et al., 1996), (iii) movement-by-deformation interactions ( $\mathrm{ezzard}$ and Clare, 1999), and (iv) movement within (as opposed to between) (which is implicitly assumed in most realignment methods, Kim et al., 1999) scans. An excellent discussion of these matters may be found in Cox (Cox, 1996. Motion and functional MRI. http://varda. biophysics.mcw.edu/ cox/regnotes.ps).

In principle it should be possible to discriminate among these causes using their expression in the temporal and spatial domain.
Interpolation effects should manifest themselves as a linear combination of periodic functions of linear combinations of the realignment parameters (Grootoonk et al., 2000), although for small movements these may be expressed as linear functions of the realignment parameters. In the spatial domain the effects should appear as linear combinations of Sinc kernels leading to the tell-tale high-frequency ringing patterns radiating from areas with large spatial derivatives.

Spin history effects would be expected, in the temporal domain, to appear as the derivative of the realignment parameters convolved with an exponential decay, whereas in the spatial domain they should contain anatomical information in that the effect should be dependent on local T1.

Movement-by-deformation interactions should be appreciable mainly in areas with nonzero deformation fields, i.e., in areas with susceptibility artefacts (for a well-shimmed scanner). Furthermore, if we 

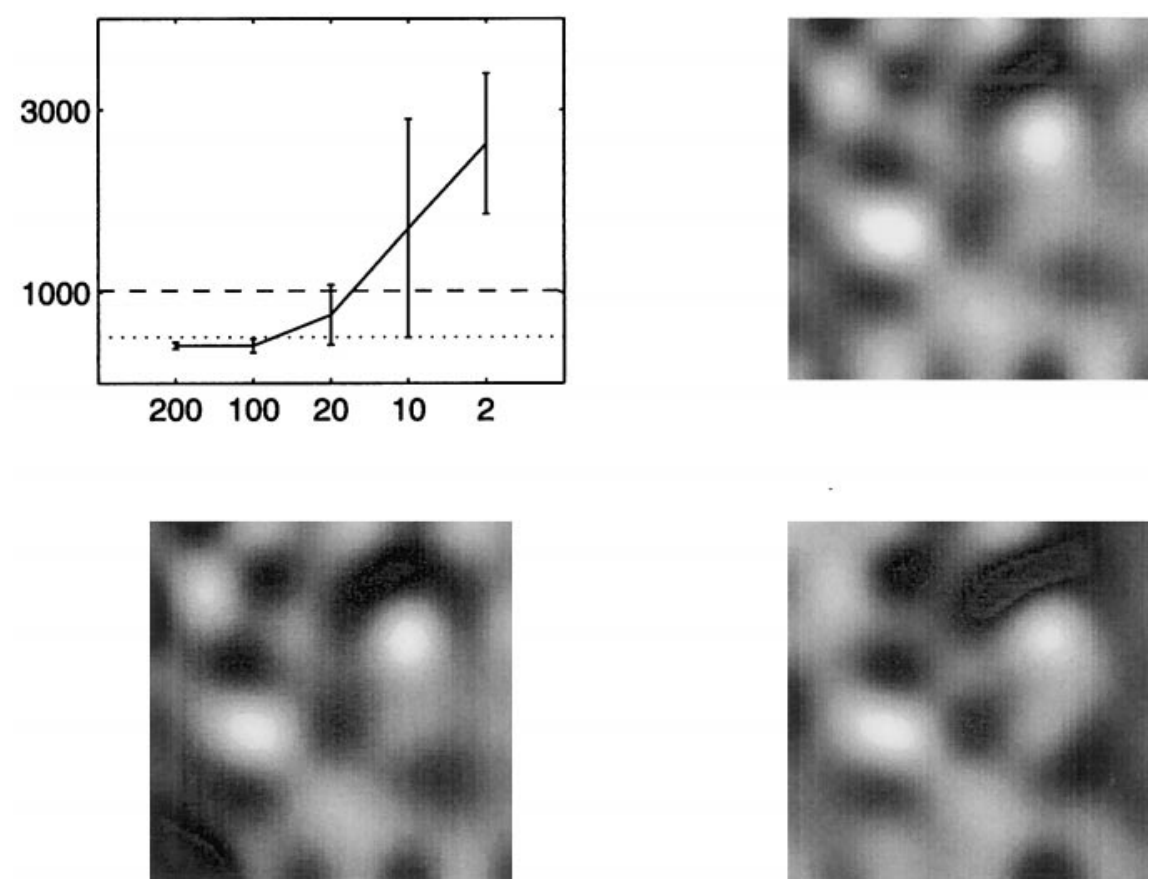

FIG. 6. Results from simulations assessing the effects of white noise on the ability to estimate the deformations. Data were generated and analyzed according to model 2. White noise was added to the data sets to yield signal-to-noise ratios (SNR) from 200 to 2 , and at each SNR 10 simulations were performed. The top left panel shows the mean and standard deviation of the sum of squared differences between the true and the estimated fields. The top right panel shows the true deformation field. The lower left and lower right panels show estimated deformation fields selected from the simulations with errors corresponding to the dotted and dashed horizontal lines in the upper left panel, respectively.

look at the series expansion suggested by Eq. (29), we would expect to find modes related to the realignment parameters (corresponding to the first partial derivatives of the deformation fields) and modes related to the realignment parameters squared (corresponding to the second partial derivatives), etc. Furthermore we would expect each subsequent mode to explain less variance due to the converging properties of the Taylor expansion, i.e., the realignment parameters should explain more variance than the realignment parameters squared. Finally it should be noted that this effect may be pronounced only for a subset of movement parameters, e.g., the out-ofplane rotations.

Finally, movement within scans should lead to apparent deformations of the object during the scan/scans within which movement occurs. In the temporal domain this should yield effects related to the temporal derivative of the movement parameters, and in the spatial domain it should be pronounced along edges in the image. The exact appearance would depend on the specific type of movement.

Hence, there is not a single cause for the movementrelated residual variance in $\mathrm{FMRI}$ time series, and it may be possible that there is no one cause that consistently dominates. It is likely that the increased use of high field scanners will render movement-by-susceptibility interactions an important source of variance. Further work in this area would ideally aim towards models accommodating all or several of these effects, since clearly there will be interactions among them. For example, high-order interpolation with Sinc kernels is implicitly based on fitting linear combinations of basis functions to the support points given by the original voxel centres. In the presence of deformation fields we mislocate the support points, yielding a different (wrong) linear combination. We suggest this as a tentative explanation for the finding $(J$. Andersson, unpublished observation) that the amount of movementrelated residual variance increases as the size of the interpolation kernel increases (the larger the kernel, the more "wrong information" is included).

In addition, all of these effects may interact with movement parameter estimation (see Andersson, 1998, for an example of interaction of interpolation errors with movement estimation). From the data collected for the present paper it is also clear that the movement-by-susceptibility interactions influence the estimation of movement parameters. When performing a reregistration of the data following "undeformation" based on the initial set of realignment parameters, we found "residual movements" in the order of $0.5^{\circ}$. Al- 
d

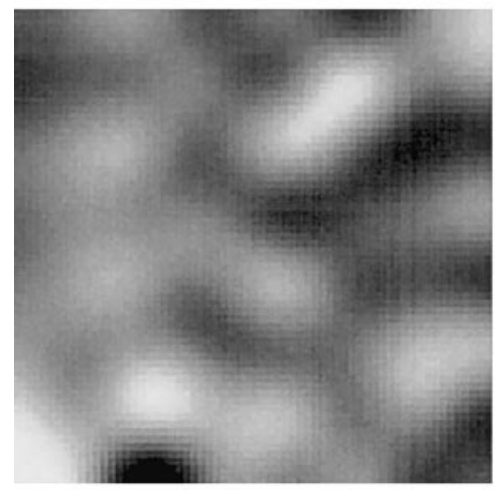

$10^{\star} \partial \mathrm{d} / \partial \mathrm{y}$

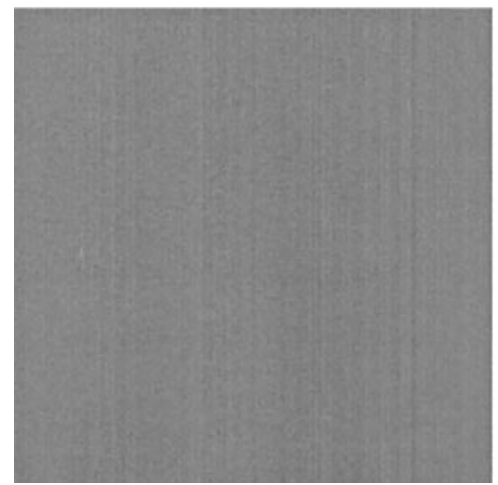

$10^{\star} \partial d / \partial x$

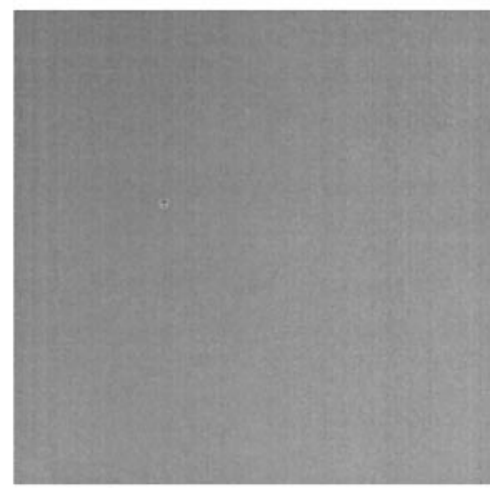

\section{$10^{\star} \partial \mathrm{d} / \partial \psi$}

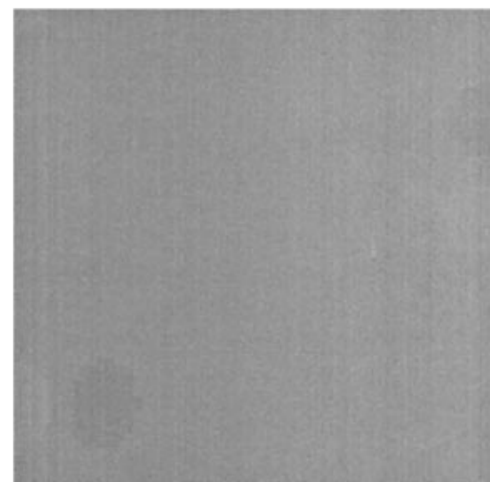

FIG. 7. Results from simulations generating data according to Eq. (3) and analyzing them using the general model defined by Eq. (28) modeling the static field and the derivatives with respect to $x$ - and y-translation and rotation $(\Psi)$. The estimated static field (upper left panel) is very similar to the "true" one (as seen, e.g., in upper left panel of Fig. 5). The derivative fields have been scaled to yield changes in deformation per $\mathrm{mm}$ or per degree for translations and rotations, respectively. The scale has been set such that the full range of the grey scale corresponds to -0.2 to 0.2 voxels, i.e., the maps are multiplied by ten compared to the static map which ranges from -2 to 2 voxels. Despite this amplification the maps show very little structure, which is consistent with them being zero as expected.

though this idea is not pursued in the present paper, it may prove beneficial to move away from the pair wise registration of images towards a "true" time-series registration in which deformation models such as those suggested here may be incorporated directly.

It is feasible to combine an explicit measurement of the deformation field at a given position when modeling the derivatives of this field according to Eq. (28). This would potentially combine the strengths of pre and post hoc methods by supplying explicit data for the field model ed by the first I columns of Eq. (28). There is potentially very little information in EPI data about the static (with respect to the object) field since only rotations around the z-axis (yaw) would be expected to contribute. There is typically little such rotation in actual time series. In addition, by modeling the derivatives there is no need to acquire a field map for each scan and there is no risk of introducing additional movement-related variance through errors in those measurements. In such a setting it might be beneficial to use a slightly more time consuming method for measuring the field map (Chen and Wyrwicz, 1999) which avoids problems with phase unwrapping.

Previous work on retrospective correction for geometric distortions in EPI images has been based on registration to images with no, or very little, geometric distortions (Studholme et al., 1999; Kybic et al., 2000). Hence, it has concentrated on the distortions per se rather than the movements-by-distortions interaction, which is the focus of the present paper. The method suggested in the present paper attempts to estimate a deformation field(s) and undistorted images of an unknown object given multiple distorted images acquired at known (or estimable) positions. In contrast, the 


\section{True d}

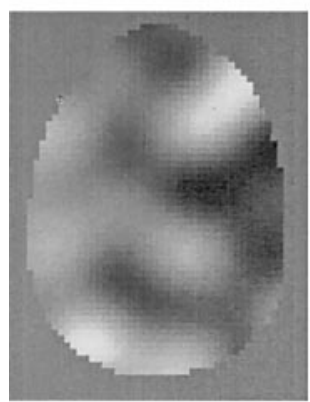

Estimated d

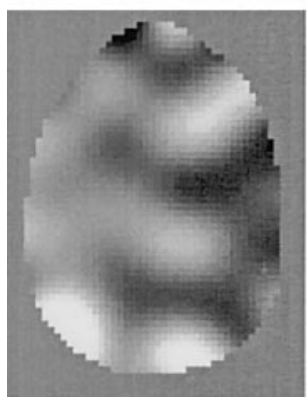

True $\partial \mathrm{d} / \partial \mathrm{x}$

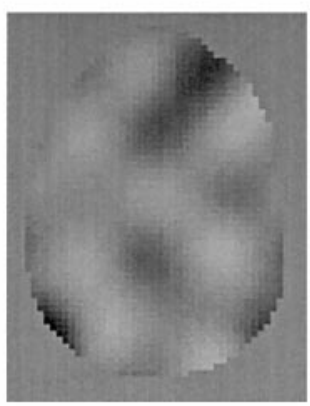

Estimated $\partial \mathrm{d} / \partial \mathrm{x}$

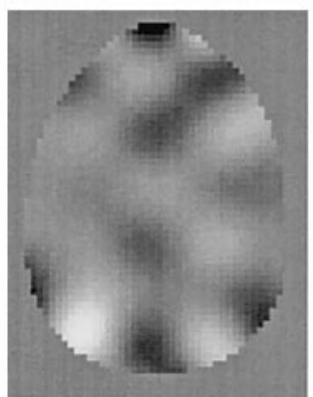

\section{True $\partial d / \partial y$}

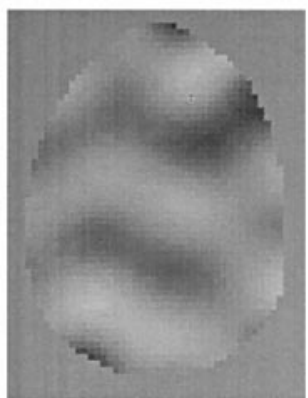

Estimated $\partial \mathrm{d} / \partial \mathrm{y}$

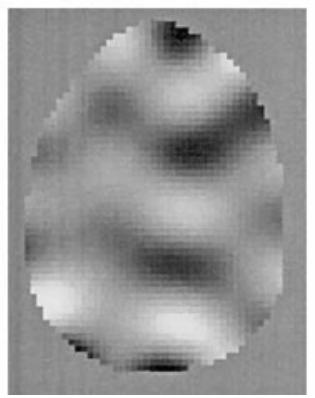

\section{True $\partial \mathrm{d} / \partial \psi$}

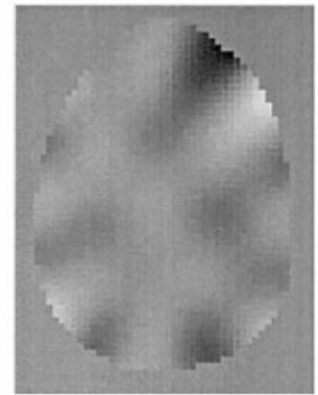

\section{Estimated $\partial \mathrm{d} / \partial \psi$}

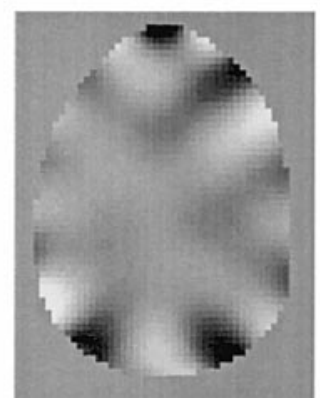

FIG. 8. True (upper row) and estimated (lower row) maps of field for object position q (column 1 ) and derivatives with respect to $x$ - and $y$-translation and rotation (columns 2 to 4 , respectively). The derivative fields are scaled to yield rate of change of deformation per millimeter and degree for translations and rotations, respectively. The derivative images are scaled such that the grey scale ranges from -0.2 to 0.2 voxels, whereas the static field ranges from -2 to 2 voxels.

other methods assume the existence of an undistorted image of the object, but with an unknown intensity mapping between them.

Further work is needed on the model we have suggested. The purpose of the present paper is to present the mathematical framework. Points that will be ad- dressed in a subsequent paper are: Which effects we need to model for EPI data, i.e., is it sufficient to model changes in deformation with respect to pitch and roll? Do we need to model some/all second order effects (i.e., Eq. (29))? How many basis functions do we need (i.e., at which spatial scales do the movement-related changes
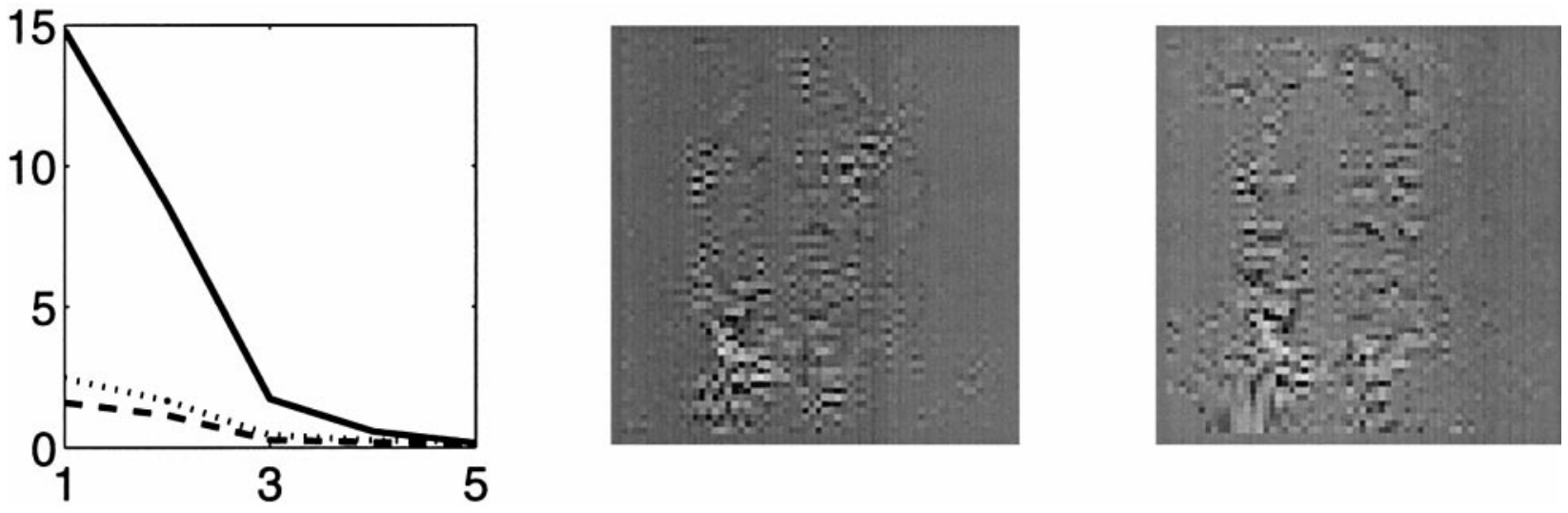

FIG. 9. The left panel shows the first five eigenvalues for the time series generated according to model 2, before "undeformation," after "undeformation" using model 2, and after "undeformation" using the general model in solid, dashed and dotted lines, respectively. The middle panel shows the first eigenimage multiplied with the first singular value after undeformation with model 2 (i.e., is identical to lower right panel of Fig. 5). The right panel shows the same after "undeformation" using the general model. 

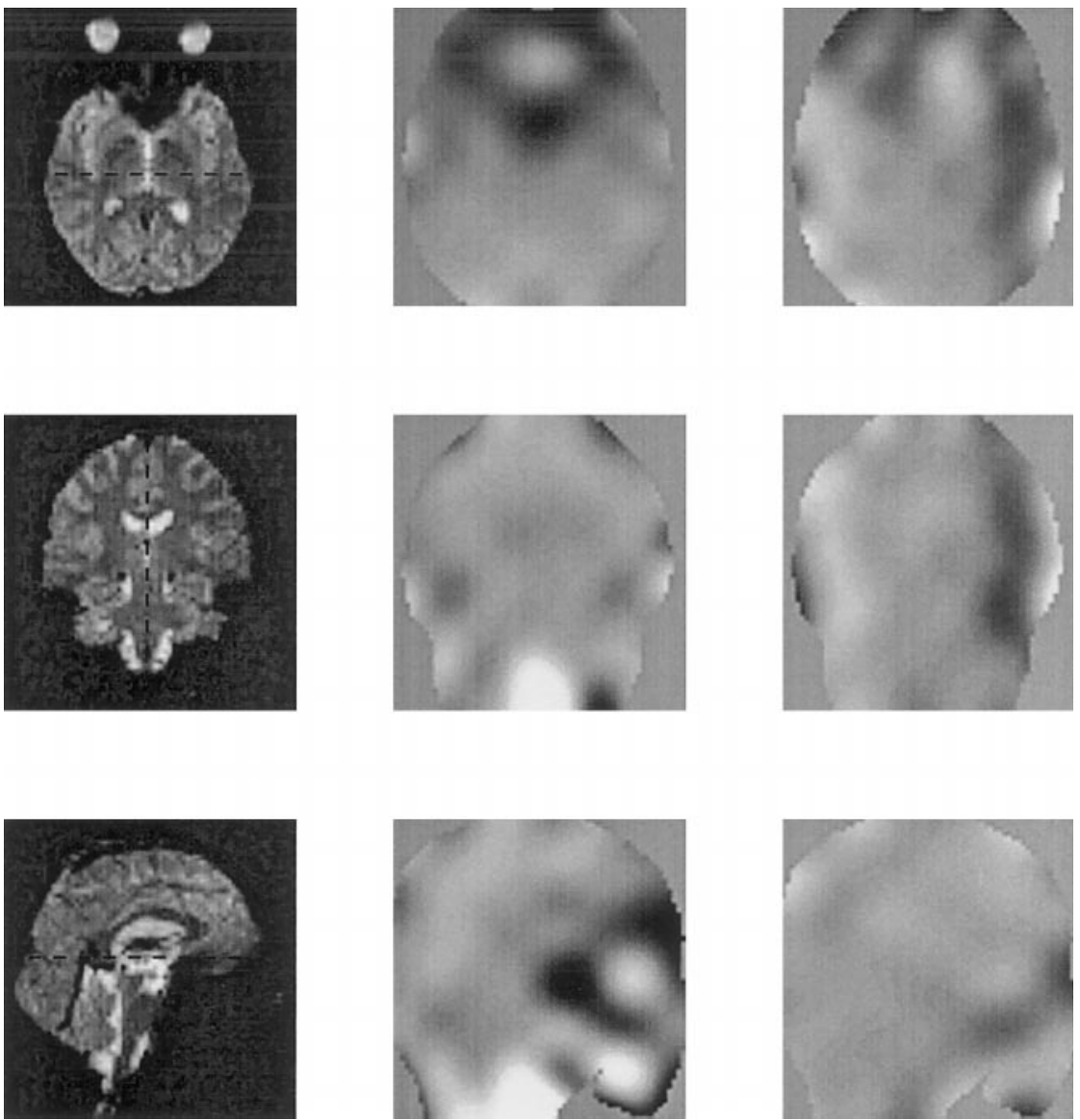

FIG. 10. The left column shows selected transversal, coronal and sagittal slices through an EPI image from the human experiment at the reference position. The middle and right columns show rate of change of deformation with respect to object rotation around the left-right $(\theta)$ and the anterior-posterior $(\phi)$ axis, respectively. The derivative images are scaled such that the range is -0.2 to 0.2 voxels per degree. Note the left-right symmetry for $\partial \mathrm{d} / \partial \theta$ and the left right antisymmetry for $\partial \mathrm{d} / \partial \phi$. Note also the relatively focal changes in areas known to be affected by susceptibility artefact such as the orbitofrontal cortex and the temporal lobes.

in resulting magnetization occur)? Can we speed the algorithm up by subsampling in the spatial and/or temporal domain? It has been shown previously that execution time for registration can be reduced by sampling only areas with high information content (Andersson, 1995), which would, in the present case, be
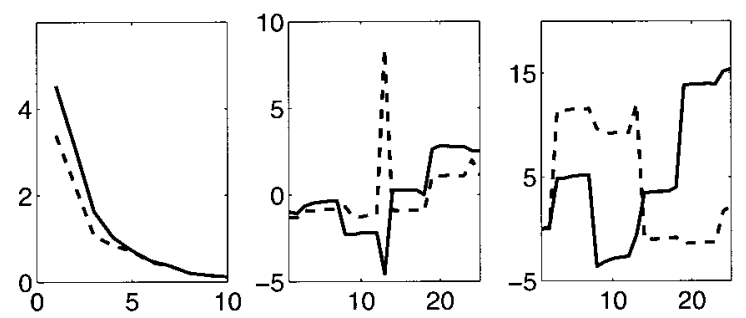

FIG. 11. Left panel shows 10 first eigenvalues for realigned (solid line) and realigned and "undeformed" (dashed line) time series. Middle panel shows first (solid line) and second (dashed line) eigenvector of realigned time series. Right panel shows estimated rotations (degrees) around the $x$ - (solid line) and y-axis (dashed line). voxels with large image gradients in images at the extremes of the estimated motion. We will also examine the method's behavior for data sets with known task-related movement.

In addition one could consider extending the model to include also the intensity correction based on the determinant of the local J acobian to compensate for the compression/dilution of signal caused by the deformations (e.g., J ezzard and Balaban, 1995; Studholme et al., 1999). The issue here is that as the deformations causes us to sample less densely than we believe, we will in addition integrate signal over a larger volume, and vice versa. Hence, in areas where the deformations change rapidly in the phase encode direction, there will in addition to the geometric deformation be an intensity scaling.

In the present paper we have presented a mathematical framework for modeling field changes resulting from subject movement. The same framework could in principle be adapted to model field changes resulting 


\section{Realigned time series Undeformed time series}
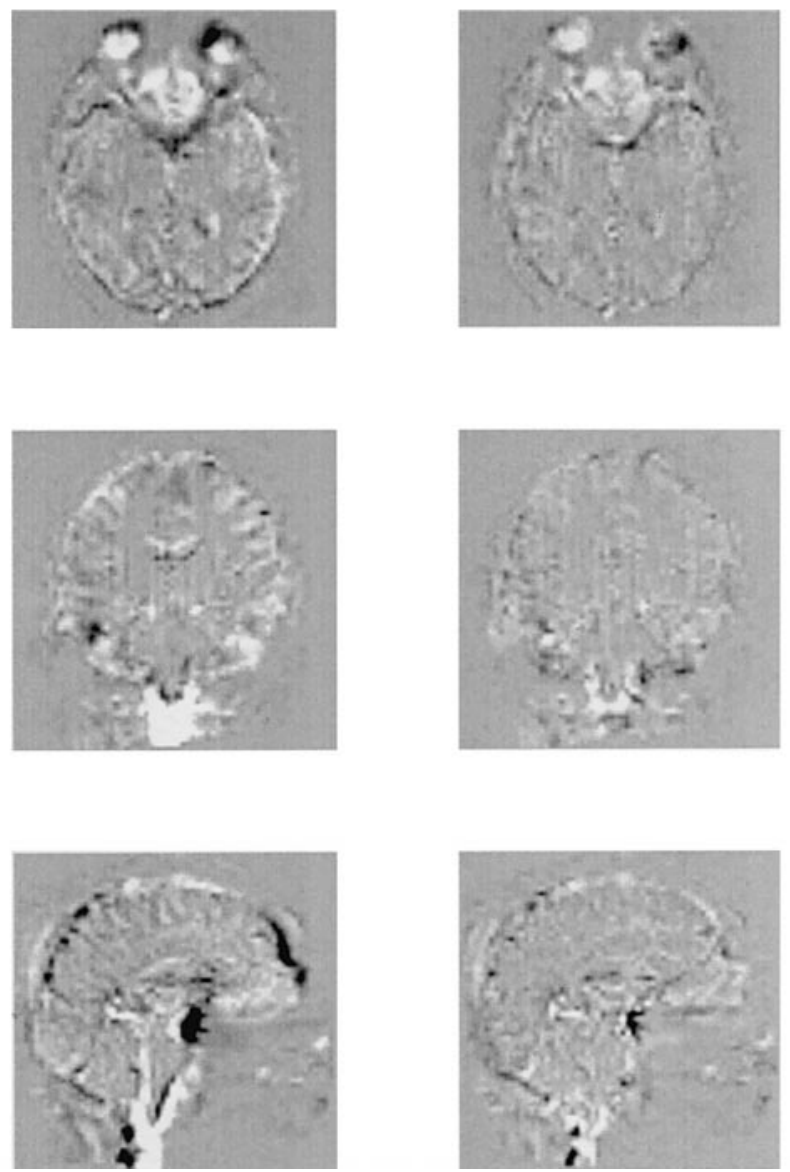

FIG. 12. First eigenimage multiplied with first singular value for realigned time series (left) and second eigenimage multiplied with second singular value for realigned and "undeformed" time series (right). The images are shown in the same scale and slices are taken at the same levels as in Fig. 9. Note how much of the movementrelated variance along the edges has been removed. This is particularly true for frontal areas as seen in the sagittal slice and temporal areas seen in the coronal slice.

from other measurable sources such as verbal responses (Birn et al., 1998). Consider a paradigm where the response could be one of a limited number of spoken words, e.g., yes or no. The resulting deformations could then be modeled using a finite impulse response (FIR) model, where a separate field is modeled following each type of response.

\section{CONCLUSION}

In the present paper we have established a mathematical framework for modeling deformations caused by magnetic field inhomogeneities. We have shown how two special cases may be modeled within the framework of a general model for which a fast algorithm exists. We have demonstrated the feasibility of the method by simulations and using an empirical data set, showing that sensible estimates of deformation fields, or derivatives thereof, are obtained and that movement-related variance can be reduced. A more comprehensive characterization of its application to EPI time-series will be reported separately.

\section{ACKNOWLEDG MENTS}

This work was supported by a grant from STiftelsen för INTernationalisering av högre utbildning och forskning (STINT) and by the Wellcome trust.

\section{REFERENCES}

Andersson, J . L. R. 1995. A rapid and accurate method to realign PET scans utilizing image edge information. J. Nucl. Med. 36: 657-669.

Andersson, J . L. R. 1998. How to obtain high-accuracy image registration: Application to movement correction of dynamic positron emission tomography data. Eur. J . Nucl. Med. 25: 575-586.

Ashburner, J ., and Friston, K. J . 1999a. I mage registration. In Functional MRI (Moonen, C. T. W., and Bandettini, P. A., Eds.), pp. 285-299. Springer-Verlag, Berlin.

Ashburner, J ., and Friston, K. J . 1999b. Nonlinear spatial normalization using basis functions. Hum. Brain Mapp. 7: 254-266.

Birn, R. M., Bandettini, P. A., Cox, R. W., J esmanowicz, A., and Shaker, R. 1998. Magnetic field changes in the human brain due to swallowing or speaking. Magn. Reson. Med. 40: 55- 60.

Chen, N.-K., and Wyrwicz, A. M. 1999. Correction for EPI distortions using multi-echo gradient-echo imaging. Magn. Reson. Med. 41: 1206-1213.

Cox, R. W., and J esmanowicz, A. 1999. Real-time 3-D image registration for functional MRI. Magn. Reson. Med. 42: 1014-1018.

Eddy, W. F., Fitzgerald, M., and Noll, D. C. 1996. Improved image registration by using F ourier interpolation. Magn. Reson. Med. 36: 923-931.

Friston, K. J ., Ashburner, J ., Frith, C. D., Poline, J .-B., Heather, J. D., and Frackowiak, R. S. J. 1995. Spatial registration and normalisation of images. Hum. Brain Mapp. 3: 165-189.

Friston, K. J ., Williams, S., Howard, R., Frackowiak, R. S. J ., and Turner, R. 1996. Movement-related effects in fMRI time-series. Magn. Reson. Med. 35: 346-355.

Grootoonk, S., Hutton, C., Ashburner, J ., Howseman, A. M., J osephs, O., Rees, G., Friston, K. J ., and Turner, R. 2000. Characterisation and correction of interpolation effects in the realignment of $\mathrm{FMRI}$ time series. Neurol mage 11: 49-57, doi:10.1006/nimg.1999.0515.

Hajnal, J . V., Myers, R., Oatridge, A., Schwieso, J . E., Young, I. R., and Bydder, G. M. 1994. Artifacts due to stimulus correlated motion in functional imaging of the brain. Magn. Reson. Med. 31: 283-291.

Hajnal, J . V., Saeed, N., Soar, E. J ., Oatridge, A., Young, I. R., and Bydder, G. M. 1995. A registration and interpolation procedure for subvoxel matching of serially acquired MR images. J . Comput. Assist. Tomogr. 19: 289-296.

J ezzard, P., and Balaban, R. S. 1995. Correction for geometric distortion in echoplanar images from $B_{0}$ field variations. Magn. Re son. Med. 34: 65-73.

J ezzard, P., and Clare, S. 1999. Sources of distortion in functional MRI data. Hum. Brain Mapp. 8: 80-85.

J iang, A., Kennedy, D. N., Baker, J . R., Weisskoff, R. M., Tootell, 
R. B. H., Woods, R. P., Benson, R. R., Kwong, K. K., Brady, T. J ., Rosen, B. R., and Belliveau, J. W. 1995. Motion detection and correction in functional MR imaging. Hum. Brain Mapp. 3: 224235.

Kim, B., Boes, J . L., Bland, P. H., Chenevert, T. L., and Meyer, C. R. 1999. Motion correction in $\mathrm{fMRI}$ via registration of individual slices into an anatomical volume. Magn. Reson. Med. 41: 964-972.

Kybic, J ., Thévenaz, P., Nirkko, A., and Unser, M. 2000. Unwarping of unidirectionally distorted EPI images. IEEE Trans. Med. Imag. 19: $80-93$.

Ostuni, J . L., Santha, A. K. S., Mattay, V. S., Weinberger, D. R., Levin, R. L., and Frank, J. A. 1997. Analysis of interpolation effects in the reslicing of functional MR images. J . Comput. Assist. Tomogr. 21: 803-810.
Reber, P. J ., Wong, E. C., Buxton, R. B., and Frank, L. R. 1998. Correction of off resonance-related distortion in echo-planar imaging using EPI-based field maps. Magn. Reson. Med. 39: 328-330.

Studholme, C., Constable, R. T., and Duncan, J . S. 1999. Incorporating an image distortion model on non-rigid alignment of EPI with conventional MRI. In Information Processing in Medical Imaging (Kuba, A., Sámal, M., and Todd-Pokropek, A., Eds.), pp. 454-459. Springer Verlag, Berlin.

Woods, R. P., Grafton, S. T., Holmes, C. J ., Cherry, S. R., and Mazziotta, J . C. 1998. Automated image registration: I. General methods and intrasubject, intramodality validation. J . Comput. Assist. Tomogr. 22: 139-152.

Wu, D. H., Lewin, J . S., and Duerk, J . L. 1997. Inadequacy of motion correction algorithms in functional MRI: Role of susceptibilityinduced artefacts. J . Magn. Reson. Imag. 7: 365-370. 